Pacific Journal of Mathematics

HARMONIC ANALYSIS ON CENTRAL HYPERGROUPS AND INDUCED RE 


\title{
HARMONIC ANALYSIS ON CENTRAL HYPERGROUPS AND INDUCED REPRESENTATIONS
}

\section{Wilfried HaUENSChILd, Eberhard KaniuTh ANd AJAy Kumar}

\begin{abstract}
Various results from harmonic analysis and representation theory for central locally compact groups and compact hypergroups, including the Plancherel theorem and the Inversion formula, as well as the finite dimensionality of irreducible representations, are extended to central hypergroups. Moreover, representations induced from unitary representations of the maximal subgroup of a hypergroup are defined and studied.
\end{abstract}

Introduction. A locally compact hypergroup $K$ is called central or a $Z$-hypergroup if $K / Z$ is compact, where $Z$ is the intersection of the maximal subgroup $G(K)$ and the center of $K$. Such hypergroups arise naturally as double coset spaces of compact subgroups of $Z$-groups (i.e. locally compact groups with cocompact center). In this paper we are primarily concerned with harmonic analysis on Z-hypergroups and their continuous irreducible representations. The basic development of harmonic analysis for hypergroups can be found in $[7,13,16,17,19]$. A survey of the subject appeared in [15].

In $\$ 1$ we prove Weil's formula and show that for arbitrary hypergroups $K$, unitary representations of subgroups of $G(K)$ can be induced up to $K$. These induced representations turn out to be very important for our investigations. Furthermore, we discuss the extension of positive definite functions. In $\$ 2$ we confine our attention to representations of $Z$-hypergroups $K$. After establishing the orthogonality relations and showing that irreducible representations are finite dimensional, we prove that the canonical map $r: \hat{K} \rightarrow \hat{Z}$ is a local homeomorphism and decompose the induced representations $U^{\lambda}, \lambda \in \hat{Z}$. Moreover, an analogue of the character formula is given. At last we apply some of our previous results to show that if $K$ is a commutative hypergroup and $H$ a subhypergroup such that $H / H \cap G(K)$ is compact, then characters of $H$ extend to characters of $K$. $\$ 3$ contains the Plancherel theorem and the Inversion formula for central hypergroups. Finally, in $\$ 4$ we briefly study representations of double coset hypergroups and present an illustrating example.

The results of $\S \S 2$ and 3 generalize the corresponding ones for central groups $[8,9,10]$ on the one hand, and most of the results of [16] and [19] for hypergroups on the other hand. We should mention that several proofs 
are similar to those in the papers just mentioned, while some of our proofs are even simpler than those given previously in the group case.

Notation and preliminaries. Throughout this paper $K$ will denote a locally compact hypergroup (same as 'convo' in [13]). The notation used is that of [13] except $\delta_{x}$ denotes the point mass at $x, x \rightarrow \check{x}$ the involution on $K$, and for a Borel function $f$ on $K$ and $x \in K,{ }_{x} f$ denotes the left translation

$$
{ }_{x} f(y)=f(x * y)=\int_{K} f(z) d \delta_{x} * \delta_{y}(z),
$$

and $f_{x}$ the right translation

$$
f_{x}(y)=f(y * x)=\int_{K} f(z) d \delta_{y} * \delta_{x}(z) .
$$

A representation $\rho$ of $K$ in the Hilbert space $H_{\rho}=H(\rho)$ is a mapping of $K$ into the algebra of bounded operators on $H_{\rho}$ such that the following hold:

(i) $x \rightarrow\langle\pi(x) u, v\rangle$ is bounded and continuous on $K$ for all $u, v \in H_{\rho}$.

(ii) $\left\langle\pi\left(\delta_{x} * \delta_{y}\right) u, v\right\rangle=\langle\pi(x) \pi(y) u, v\rangle$ and $\pi(\check{x})=\pi(x)^{*}$ for all $x, y$ $\in K$ and $u, v \in H_{\rho}$.

$$
\langle\pi(\mu) u, v\rangle=\int_{K}\langle\pi(x) u, v\rangle d \mu(x), \quad \mu \in M(K),
$$

is a representation of $K$ in the sense of $[13,11.3]$ in case $\pi$ is non-degenerate, since by $[13,2.2$.D $]$ it follows that $\mu \rightarrow\langle\pi(\mu) u, v\rangle$ is continuous on $M^{+}(K)$. The notion of equivalence between representations is that of unitary equivalence.

We shall denote the maximal subgroup of $K$ by

$$
G(K)=\left\{x \in K ; \delta_{x} * \delta_{\check{x}}=\delta_{e}\right\},
$$

and the center of $K$ by

$$
Z(K)=\left\{x \in K ; \delta_{x} * \delta_{y}=\delta_{y} * \delta_{x} \text { for all } y \in K\right\} .
$$

For a subhypergroup $H$ of $K, K / H=\{\dot{x}=x * H ; x \in K\}$ is equipped with the quotient topology, so that the natural projection $p: K \rightarrow K / H$ is open and continuous. For a normal subgroup $H$ of $K$ (i.e. a subgroup of $G(K)$ which is normal in the sense that $x * H=H * x$ for all $x \in K)$ it is easy to show that $K / H$ is a locally compact hypergroup under the convolution defined by

$$
\int_{K / H} f(\dot{z}) d \delta_{\dot{x}} * \delta_{\dot{y}}(\dot{z})=\int_{K} f \circ p(z) d \delta_{x} * \delta_{y}(z)
$$


for all $f \in C_{00}(K / H)$ and $x, y \in K$. For $x \in K$ and $t \in G(K)$, we will write $x t$ for the unique element $y$ satisfying $\delta_{x} * \delta_{t}=\delta_{y}$.

If $K$ has a left Haar measure, then $L^{1}(K)$ denotes the convolution algebra. For $f \in L^{1}(K), x \rightarrow{ }_{x} f$ is continuous from $K$ into $L^{1}(K)[13$, 2.2.B, 5.4.H]. It is easy to show that $L^{1}(K)$ has a bounded approximate identity $\left\{u_{i} ; i \in I\right\} \subseteq C_{00}^{+}(K)$ such that $\left\|u_{i}\right\|_{1}=1$. Hence we can form the enveloping $C^{*}$-algebra $C^{*}(K)$ of $L^{1}(K)[5, \S 2.7]$. Since the left regular representation of $L^{1}(K)$ on $L^{2}(K)$ is faithful, the map $L^{1}(K) \rightarrow C^{*}(K)$ is injective. Finally, $\hat{K}=\widehat{C^{*}(K)}$ denotes the dual space of $K\left(\operatorname{resp} . C^{*}(K)\right)$ endowed with the usual topology $[5, \S 3.1]$.

1. Weil's formula and induced representations. We didn't succeed in defining induced representations in the case of an arbitrary locally compact hypergroup $K$ and a closed subhypergroup $H$ of $K$. But the main purpose of this section is to show that when $H$ is a subgroup of $K$, then unitary representations of $H$ can be induced up to $K$. This will be done by modifying Blattner's proof [1] for the group case.

LEMMA 1.1. Let $H$ be a subhypergroup of $K$ possessing a left Haar measure. For $f \in C_{00}(K)$, let $T_{H} f$ on $K / H$ be defined by

$$
T_{H} f(\dot{x})=\int_{H} f(x * t) d t .
$$

Then $T_{H}$ is a linear mapping from $C_{00}(K)$ onto $C_{00}(K / H)$. Moreover, $C_{00}^{+}(K / H)=T_{H}\left(C_{00}^{+}(K)\right)$.

Proof. $T_{H}(f)$ is well defined since $x \rightarrow \int_{H} f(x * t) d t$ is constant on left cosets of $H$. Let $x \in K$ and $\varepsilon>0$ be given, and choose a compact neighborhood $U_{0}$ of $x$ in $K$. Then $C=\left(\check{U}_{0} * \operatorname{supp} f\right) \cap H$ is a compact subset of $H$ with finite measure $M$. Because of the continuity of $(x, y) \rightarrow$ $f(x * y)$ there exists a neighborhood $U \subseteq U_{0}$ of $x$ such that $\left\|_{y} f-{ }_{x} f\right\|_{\infty}<$ $M^{-1} \varepsilon$ for all $y \in U$. On the other hand, $f(y * t) \neq 0$ for some $t \in H$ implies $\left(U_{0} * t\right) \cap \operatorname{supp} f \neq \varnothing$, hence $t \in\left(\check{U}_{0} * \operatorname{supp} f\right) \cap H=C$ [13, Lemma 4.1.B]. Thus

$$
\left|T_{H} f(\dot{y})-T_{H} f(\dot{x})\right| \leq \int_{C}|f(y * t)-f(x * t)| d t<\varepsilon
$$

for all $y \in U$. Clearly, $T_{H}$ is a linear mapping into $C_{00}(K / H)$. Given $g \in C_{00}^{+}(K / H)$, the existence of an $f \in C_{00}^{+}(K)$, such that $T_{H} f=g$, can be proved as in the group case [14, Ch. $3, \S 4.2]$.

Let $H$ be a subhypergroup of $K$ and suppose that $K$ and $H$ possess left Haar measures with modular functions $\Delta_{K}$ and $\Delta_{H}$, respectively. Set 
$\delta(t)=\Delta_{H}(t)^{1 / 2} \Delta_{K}(t)^{-1 / 2}$ for $t \in H$. We are looking for solutions $\varphi$, in particular strictly positive, continuous ones, of the following equation:

$$
\varphi(x * t)=\delta(t)^{2} \varphi(x) \text { for } x \in K, t \in H .
$$

The proof the following lemma is a slight modification of the one in $[14$, Ch. 8, §1.7].

LEMMA 1.2. Let $H$ be a subhypergroup of $K$. There exists a non-negative continuous function $F$ on $K$ satisfying

(i) For every $x \in K$ there is a $t \in H$ such that $F(x * t)>0$.

(ii) If $W \subseteq K$ is compact, then $F$ coincides on $W * H$ with some function in $C_{00}(K)$.

Proof. Let $x \in K$ and $U$ be an open relatively compact neighborhood of $e$ in $K$. Let $C=(U \cup \check{U})^{-}$and $L=\cup_{n=1}^{\infty} C^{n}$; then $L$ is an open subhypergroup of $K$. Replacing $\Omega$ by $U, G_{1}$ by $L * x$ and $K^{r}$ by $C^{r} * x$ in the proof of $[14, \mathrm{Ch} .8, \S 1.7]$, we obtain a subset $Y_{x}$ of $L * x$ such that $\left\{U * y * H ; y \in Y_{x}\right\}$ is an open locally finite covering of $L * x * H$. Consider the double cosets $L * x * H, x \in K$. They are open and pairwise disjoint or equal (the proof in [13, (14.1.A)] does not use $L=H$ ). Choose $A \subseteq K$ such that $K=\cup_{x \in A} L * x * H$ is a disjoint union, and set $Y=$ $\cup_{x \in A} Y_{x}$. Then $\{U * x * H ; x \in Y\}$ is an open, locally finite covering of $K$. Take now any $f \in C_{00}^{+}(K)$ such that $f(e)>0$ and apply the above to $U=\{x \in K ; f(x)>0\}$. Since $U * y=\left\{x \in K ; f_{y}(x)>0\right\}$ [13, (4.2.D)], $F=\Sigma_{y \in Y} f_{\check{y}}$ defines a continuous function on $K$. If $W \subseteq K$ is compact, then there are only finitely many $y \in Y$ such that $W * H \cap U * y \neq \varnothing$, so (ii) holds. Finally, if $x \in K$, then $x \in U * y * H$ for some $y \in Y$, hence $x * H \cap U * y \neq \varnothing$, and this implies $F(x * t) \geq f_{\mathfrak{y}}(x * t)>0$ for some $t \in H$.

COROLlaRY 1.3. Let $H$ be a subhypergroup of $K$ and suppose that $K$ and $H$ admit left Haar measures. Then there exists a strictly positive, continuous function $q$ on $K$ satisfying equation (q).

Proof. Set $q(x)=\Delta_{K}(x)^{-1} \int_{H} F(x * t) d t$.

REMARK. We will always choose $q=1$ in case $\Delta_{H}=\Delta_{K} \mid H$.

Let's now turn to the case where $H$ is a subgroup of $K$ and consider any locally integrable function $\varphi: K \rightarrow \mathbf{C}$ satisfying (q); we shall call such 
a function a $q$-function. Then for $f, g \in C_{00}(K)$ the following holds:

$$
\begin{aligned}
\int_{K} f(x) T_{H} g(\dot{x}) \varphi(x) d x & =\int_{K} \int_{H} f(x) g(x t) \varphi(x) d t d x \\
& =\int_{H} \int_{K} \Delta_{K}(\check{t}) f(x \check{t}) g(x) \varphi(x \check{t}) d x d t \\
& =\int_{K} \int_{H} \Delta_{H}(\check{t}) f(x \check{t}) g(x) \varphi(x) d t d x \\
& =\int_{K} T_{H} f(\dot{x}) g(x) \varphi(x) d x .
\end{aligned}
$$

We would like to mention that a similar computation holds in case $H$ is a subhypergroup of $K, H$ and $K$ being unimodular and $\varphi=1$.

Now given $f$ we can choose $g \in C_{00}(K)$ such that $T_{H} g \mid p(\operatorname{supp} f)=1$; then $T_{H} f=0$ implies $\int_{K} f(x) \varphi(x) d x=0$. Thus by

$$
\mu_{\varphi}\left(T_{H} f\right)=\int_{K} f(x) \varphi(x) d x,
$$

a linear functional on $C_{00}(K / H)$ can be defined. For $\varphi \geq 0$ we get a positive (possibly unbounded) measure on $K / H$. If $\varphi$ is an arbitrary $q$-function, then so is $|\varphi|$; hence $\mu_{\varphi}$ is a complex measure in case $\mu_{|\varphi|}$ is bounded.

Applying this to the function $q$ of Corollary 1.3 we get

LEMMA 1.4. Let $K$ be a hypergroup with left Haar measure and $H$ a subgroup of $K$. Then a positive measure $d_{q}$ on $K / H$ can be chosen such that

$$
\int_{K / H}\left(\int_{H} f(x t) d t\right) d_{q} \dot{x}=\int_{K} f(x) q(x) d x \quad \text { for } f \in C_{00}(K) .
$$

REMARK 1.5. Given $K, H$ and $q$ we can consider $T_{H, q}: C_{00}(K) \rightarrow$ $C_{00}(K / H)$ defined by $T_{H, q} f=T_{H}\left(f \cdot q^{-1}\right) . T_{H, q}$ again is a linear surjection, by the aid of which we may write Weil's formula in the following way:

$$
\int_{K / H} T_{H, q} f(\dot{x}) d_{q} \dot{x}=\int_{K} f(x) d x \quad \text { for } f \in C_{00}(K) .
$$

As in [14, Ch. 3, 4.5 and Ch. 8, 2.3] we can extend these two formulas to integrable functions: (i) For $f \in L^{1}(K)$ there is a null set $A_{0}$ in $K / H$ such that $t \rightarrow f(x t) \in L^{1}(H)$ for every $x \notin p^{-1}\left(A_{0}\right)$. (ii) The function $\dot{x} \rightarrow \int_{H} f(x t) d t$, defined almost everywhere, is in $L^{1}(K / H)$. 
(iii)

$$
\int_{K / H}\left(\int_{H} f(x t) d t\right) d_{q} \dot{x}=\int_{K} f(x) q(x) d x .
$$

If $\Delta_{H}=\Delta_{K} \mid H$ holds, we take $q=1$ and write $d \dot{x}$ instead of $d_{q} \dot{x}$. Now we give a generalization of [13, Theorem 5.1.D] which will be important later on.

LEMMA 1.6. Let $K$ and $H$ be as in Lemma 1.4 with $\Delta_{H}=\Delta_{K} \mid H$. Assume further that $f$ and $g$ are continuous functions on $K$ with compact support modulo $H$ such that

$$
f(z t) g(x t)=f(z) g(x) \text { for } x, z \in K \text { and } t \in H \text {. }
$$

Then for $y \in K$ the functions $x \rightarrow f(y * x) g(x)$ and $x \rightarrow f(x) g(\check{y} * x)$ are constant on cosets of $H$, and

$$
\int_{K / H} f(y * x) g(x) d \dot{x}=\int_{K / H} f(x) g(\check{y} * x) d \dot{x} .
$$

Proof. Both functions are supported by some compact subset $C$ of $K / H$. Using [13, Theorem 5.1.D] and selecting $h \in C_{00}(K)$ such that $T_{H} h \mid C=1$, we get

$$
\begin{aligned}
\int_{K / H} f(x) g( & \check{y} * x) d \dot{x}=\int_{K}(h \cdot f)(x) g(\check{y} * x) d x \\
& =\int_{K}(h \cdot f)(y * x) g(x) d x=\int_{K / H} \int_{H}(h \cdot f)(x t) g(x t) d t d \dot{x} \\
& =\int_{K / H} \int_{K} \int_{H} h(z t) f(z t) g(x t) d \delta_{y} * \delta_{x}(z) d t d \dot{x} \\
& =\int_{K / H} \int_{p^{-1}(C)}\left(\int_{H} h(z t) d t\right) f(z) g(x) d \delta_{y} * \delta_{x}(z) d \dot{x} \\
& =\int_{K / H} f(y * x) g(x) d \dot{x} .
\end{aligned}
$$

REMARK 1.7. It follows from the remark preceding Lemma 1.4 that 1.4-1.6 are valid also for a subhypergroup $H$ possessing a left Haar measure provided that $H$ and $K$ are unimodular.

Our next lemma deals with the relationship of left Haar measures on $K$ and $K / H$. 
LEMMA 1.8. Let $H$ be a normal subgroup of $K$. Then $K$ admits a left Haar measure such that $\Delta_{H}=\Delta_{K} \mid H$ iff $K / H$ has a Haar measure.

Proof. If $d x$ is a left Haar measure on $K$ such that $\Delta_{H}=\Delta_{K} \mid H$, then 1 is a $q$-function and a left Haar measure on $K / H$ can be defined by $\int_{K / H} T_{H} f(\dot{x}) d \dot{x}=\int_{K} f(x) d x$. Conversely, if $d \dot{x}$ exists, then $f \rightarrow$ $\int_{K / H} T_{H} f(\dot{x}) d \dot{x}$ defines a Haar measure on $K$. Moreover, $\Delta_{K} \mid H=\Delta_{H}$ since

$$
\begin{aligned}
\Delta_{K}(s) \int_{K} f(x) d x & =\int_{K} f(x \check{s}) d x=\int_{K / H} \int_{H} f(x t \check{s}) d t d \dot{x} \\
& =\Delta_{H}(s) \int_{K} f(x) d x
\end{aligned}
$$

for all $f \in C_{00}(K)$ and $s \in H$.

Now we are going to define induced representations. So we are given a hypergroup $K$ with left Haar measure, a subgroup $H$ of $K$ and a unitary representation $\rho$ of $H$ in a Hilbert space $H_{\rho}$. Let $\mathscr{F}^{\prime}$ denote the linear space of all mappings $\xi: K \rightarrow H_{\rho}$ having the following properties:

(i) $\xi$ is Bourbaki measurable.

(ii) $\xi(x t)=\delta(t) \rho(\check{t}) \xi(x)$ for $x \in K$ and $t \in H$.

(iii) $x \rightarrow\|\xi(x)\|^{2}$ is locally integrable.

(ii) forces $x \rightarrow\langle\xi(x), \eta(x)\rangle, \xi, \eta \in \mathscr{F}^{\prime}$, to be a $q$-function, since $\rho$ is unitary. Therefore a positive measure $\mu_{\xi}$ on $K / H$ can be defined by $\mu_{\xi}\left(T_{H} f\right)=\int_{K} f(x)\|\xi(x)\|^{2} d x, f \in C_{00}(K)$. Clearly, $\mathscr{F}=\left\{\xi \in \mathscr{F}^{\prime} ; \mu_{\xi}\right.$ is bounded $\}$ is a linear subspace of $\mathscr{F}^{\prime}$, and $\xi, \eta \in \mathscr{F}$ define a complex measure $\mu_{\xi, \eta}$ on $K / H$ by $\mu_{\xi, \eta}\left(T_{H} f\right)=\int_{K} f(x)\langle\xi(x), \eta(x)\rangle d x$. We obtain a positive semidefinite hermitian form $\langle\xi, \eta\rangle=\mu_{\xi, \eta}(K / H)$ on $\mathscr{F}$, and setting $\mathscr{T}=\left\{\xi \in \mathscr{F} ; \mu_{\xi}=0\right\}$, one proves as in [1, Lemma 1 and Proposition 1] that $\mathscr{K}=\mathscr{F} / \mathcal{K}$ is a Hilbert space. Following [1] denote by $\mathscr{F}_{0}$ the subspace of $\mathscr{F}^{\prime}$ consisting of continuous mappings having compact support modulo $H$. If the support of $\xi \in \mathscr{F}_{0}$ is contained in $p^{-1}(C)$ for some compact subset $C$ of $K / H$, then supp $\mu_{\xi} \subseteq C$, hence $\xi \in \mathscr{F}$. For $f \in C_{00}(K)$ and $v \in H_{\rho}$ set

$$
\varepsilon(f, v)(x)=\int_{H} \delta(t)^{-1} f(x t) \rho(t) v d t
$$

Then $\varepsilon$ is a bilinear mapping from $C_{00}(K) \times H_{\rho}$ into $\mathscr{F}_{0}$, and for $\xi=\varepsilon(f, v)$ and $\eta \in \mathscr{F}$, supp $\mu_{\xi}$, as well as $\operatorname{supp} \mu_{\xi, \eta}$, is contained in $p(\operatorname{supp} f)$. Since 
$H$ is a group, one gets, as in [1],

$$
\langle\varepsilon(f, v), \eta\rangle=\int_{K} f(x)\langle v, \eta(x)\rangle d x .
$$

Thus we obtain [1, Lemma 2]:

(i) $\|\varepsilon(f, v)\| \leq \Lambda_{\text {supp } f}\|f\|_{\infty}\|v\|$, where $\Lambda_{\text {supp } f}$ depends only on the compact set $\operatorname{supp} f$. in $\mathscr{K}$.

(ii) If $D$ is total in $H_{\rho}$, then $\tilde{D}=\left\{\varepsilon(f, v) ; f \in C_{00}(K), v \in D\right\}$ is total

The difficulties arising from the fact that $K$ is not necessarily a group, are extracted in the following

LEMMA 1.9. (i) ${ }_{y} \varepsilon(f, v)=\varepsilon\left({ }_{y} f, v\right)$ for $f \in C_{00}(K), v \in H_{\rho}$ and $y \in K$.

(ii) If $\xi \in \mathscr{F}_{0}$ such that ${ }_{y} \xi \in \mathscr{F}_{0}$, then $\left\|_{y} \xi\right\| \leq\|\xi\|$.

Proof. (i) Put $C=\operatorname{supp} f$ and choose $g \in C_{00}(K)$ such that $T_{H} g$ $p(\check{y} * C)=1$. Since, for every $\eta \in \mathcal{F}, \operatorname{supp} \mu_{y \varepsilon(f, v), \eta}$ and $\operatorname{supp} \mu_{\varepsilon(y, f), \eta}$ are contained in $p(\check{y} * C)$, we get

$$
\begin{aligned}
\left\langle{ }_{y} \varepsilon(f, v), \eta\right\rangle & =\int_{K} g(x)\left\langle_{y} \varepsilon(f, v)(x), \eta(x)\right\rangle d x \\
& =\int_{K} g(x) \int_{K} \int_{H} \delta(t)^{-1} f(z t)\langle\rho(t) v, \eta(x)\rangle d t d \delta_{y} * \delta_{x}(z) d x \\
& =\int_{K} g(x) \int_{H} \delta(t)^{-1}{ }_{y} f(x t)\langle\rho(t) v, \eta(x)\rangle d t d x \\
& =\int_{K} g(x)\left\langle\varepsilon\left({ }_{y} f, v\right)(x), \eta(x)\right\rangle d x=\left\langle\varepsilon\left({ }_{y} f, v\right), \eta\right\rangle .
\end{aligned}
$$

(ii) Let $\xi \in \mathscr{F}_{0}$ such that ${ }_{y} \xi \in \mathscr{F}_{0}$ and select $g \in C_{00}^{+}(K)$ so that $T_{H} g \mid \operatorname{supp} \mu_{y \xi}=1$ and $\left\|T_{H} g\right\|_{\infty}=1$. Then

$$
\begin{aligned}
\left\|_{y} \xi(x)\right\| & \leq \sup \left\{\int_{K}|\langle\xi(z), v\rangle| d \delta_{y} * \delta_{x}(z) ; v \in H_{\rho},\|v\| \leq 1\right\} \\
& \leq \int_{K}\|\xi(z)\| d \delta_{y} * \delta_{x}(z) \leq\left(\int_{K}\|\xi(z)\|^{2} d \delta_{y} * \delta_{x}(z)\right)^{1 / 2} ;
\end{aligned}
$$

hence

$$
\begin{aligned}
\left\|_{y} \xi\right\|^{2} & =\int_{K} g(x)\left\|_{y} \xi(x)\right\|^{2} d x \leq \int_{K} g(x)\left(\int_{K}\|\xi(z)\|^{2} d \delta_{y} * \delta_{x}(z)\right) d x \\
& =\int_{K} g(\check{y} * x)\|\xi(x)\|^{2} d x=\int_{K / H} T_{H}(\check{y} g)(\dot{x}) d \mu_{\xi}(\dot{x}) .
\end{aligned}
$$


Though $H$ is not necessarily normal, we nevertheless have

$$
T_{H}\left({ }_{y} g\right)(\dot{x})=\int_{K} T_{H} g(\dot{z}) d \delta_{\check{y}} * \delta_{x}(z) \leq\left\|T_{H} g\right\|_{\infty} .
$$

Thus we obtain

$$
\|\|_{y}\left\|^{2} \leq \int_{K / H} T_{H}(\dot{y} g)(\dot{x}) d \mu_{\xi}(\dot{x}) \leq\right\| \mu_{\xi}\|=\| \xi \|^{2} .
$$

Now we are ready to define the induced representation. $\xi \rightarrow_{y} \xi$ is a bounded linear mapping from a dense subspace of $\mathscr{K}$ into itself, namely the subspace generated by the $\varepsilon(f, v), v \in H_{\rho}, f \in C_{00}(K)$. Hence it can be extended to a bounded linear operator $U^{\rho}(y)$ on $\mathscr{K}$.

Proposition 1.10. $U^{\rho}: y \rightarrow U^{\rho}(y)$ is a continuous representation of $K$ in $\mathcal{K}$.

Proof. Given $\varepsilon(f, v), \eta \in \mathscr{F}_{0}$ and $x \in K$, choose a compact neighborhood $U_{0}$ of $x$. Then $|\langle v, \eta(z)\rangle| \leq M<\infty$ for every $z \in U_{0} * \operatorname{supp} f$ and a suitable $M$. Now there exists a neighborhood $U \subseteq U_{0}$ of $x$ such that $\left\|_{\check{y}} f-_{\check{x}} f\right\|<\varepsilon / M$ for all $y \in U$. Thus

$$
\begin{aligned}
\mid\left\langle\left( U^{\rho}(y)\right.\right. & \left.\left.-U^{\rho}(x)\right) \varepsilon(f, v), \eta\right\rangle \mid \\
\leq & \int_{K}\left\|_{\breve{y}} f(z)-{ }_{\check{x}} f(z)\right\| \cdot|\langle v, \eta(z)\rangle| d z \leq \varepsilon .
\end{aligned}
$$

By Lemma 1.9(ii) we have $\left\|U^{\rho}(y)\right\| \leq 1$. For $\varepsilon(f, v), \eta \in \mathscr{K}$ and $x, y \in K$ we have

$$
\begin{aligned}
\int_{K}\left\langle U^{\rho}(z) \varepsilon\right. & (f, v), \eta\rangle d \delta_{x} * \delta_{y}(z) \\
& =\int_{K} \int_{K} f(\check{z} * w)\langle v, \eta(w)\rangle d w d \delta_{x} * \delta_{y}(z) \\
& =\int_{K} f(\check{y} * \check{x} * w)\langle v, \eta(w)\rangle d w=\left\langle\varepsilon\left(_{\check{x}}(\check{y} f), v\right), \eta\right\rangle,
\end{aligned}
$$

i.e. $U^{\rho}(x * y)=U^{\rho}(x) U^{\rho}(y)$. Similarly, one verifies

$$
\left\langle U^{\rho}(\check{x}) \varepsilon(f, v), \varepsilon(g, u)\right\rangle=\left\langle\varepsilon(f, v), U^{\rho}(x) \varepsilon(g, u)\right\rangle,
$$

i.e. $U^{\rho}(\check{x})=U^{\rho}(x)^{*}$. (Concerning the associativity see $[13, \S 3]$.)

We call $U^{\rho}$ the representation induced by $\rho$ and henceforth denote $\mathscr{K}$ by $H\left(U^{\rho}\right)$. 
We next give Blattner's description of the induced representation in terms of positive definite measures [2] in our setting. This will turn out to be very useful.

Let $\mu$ be a positive definite measure on the hypergroup $K$, i.e. $\mu\left(f^{*} * f\right) \geq 0$ for all $f \in C_{00}(K)$, and set $N^{\mu}=\left\{f \in C_{00}(K) ; \mu\left(f^{*} * f\right)\right.$ $=0\}$. Then $N^{\mu}$ is a left ideal and left translation invariant, and $L^{\mu}=$ $C_{00}(K) / N^{\mu}$ is a pre-Hilbert space with inner product $\left\langle f^{\mu}, g^{\mu}\right\rangle=$ $\mu\left(g^{*} * f\right)$. Every $x \in K$ defines a linear operator $V^{\mu}(x)$ in $L^{\mu}$ by $V^{\mu}(x) f^{\mu}$ $=\left({ }_{\check{x}} f\right)^{\mu}$. We assume now that $K$ has a left Haar measure and denote by $P(K)$ the set of all bounded continuous positive definite functions on $K$. Let $\varphi \in P(K), N^{\varphi}=\left\{f \in C_{00}(K) ;\left\langle f^{*} * f, \varphi\right\rangle=0\right\}$ and $H^{\varphi}$ be the completion of $C_{00}(K) / N^{\varphi}$ with respect to the inner product $\left\langle f^{\varphi}, g^{\varphi}\right\rangle=$ $\left\langle g^{*} * f, \varphi\right\rangle, f^{\varphi}=f+N^{\varphi}$. $\varphi$ defines a cyclic representation $\pi_{\varphi}$ of $K$ on $H^{\varphi}$ such that $\pi_{\varphi}(x) f^{\varphi}=\left({ }_{\check{x}} f\right)^{\varphi}$ and $\varphi(x)=\left\langle\pi_{\varphi}(x) v, v\right\rangle$ for some cyclic vector $v$. Moreover, $\pi_{\varphi}$ is irreducible iff $\varphi$ is indecomposable (same as irreducible in $[13, \S 11])$.

Suppose now that $H$ is a subgroup of $K$ and define $R: C_{00}(K) \rightarrow$ $C_{00}(H)$ by $R(f)(t)=\delta(\check{t}) f(t) . \varphi$ defines a measure $\mu_{\varphi}$ on $K$ by $\mu_{\varphi}(f)=$ $\int_{H} \varphi(t) R(f)(t) d t$.

Given $g, f \in C_{00}(K)$, choose $h \in C_{00}^{+}(K)$ such that $\int_{H} h(x t) d t=1$ for all $x \in \operatorname{supp} f \cup \operatorname{supp} g$ (Lemma 1.1). Using the fact that $H \subseteq G(K)$, by a lengthy, but straightforward, computation one verifies the following formula:

$$
\mu_{\varphi}\left(f^{*} * g\right)=\int_{K} h(x)\left(\int_{H} \int_{H} \overline{R\left({ }_{x} f\right)(t)} R\left({ }_{x} g\right)(s) \varphi(\check{t} s) d s d t\right) d x .
$$

(Compare the proof of [2, Theorem 1].) Thus $\mu_{\varphi}$ is positive definite. The easiest way to show that $x \rightarrow V^{\mu_{\varphi}}(x)$ defines a representation of $K$, which is in fact equivalent to $U^{\pi_{\varphi}}$, is now to show:

LEMMA 1.11. With the above assumptions and notations a linear mapping $\phi: L^{\mu_{\varphi}} \rightarrow H\left(U^{\pi_{\varphi}}\right)$ can be defined by $\phi\left(f^{\mu_{\varphi}}\right)(x)=R\left({ }_{x} f\right)^{\varphi} . \phi$ is isometric and dense and satisfies $\phi V^{\mu_{\varphi}}(x)=U^{\pi_{\varphi}}(x) \phi$ for all $x \in K$. Hence $V^{\mu_{\varphi}}(x)$ extends to a bounded linear operator on $H^{\mu_{\varphi}}$, the completion of $L^{\mu_{\varphi}}$, and $V^{\mu_{\varphi}}: x \rightarrow V^{\mu_{\varphi}}(x)$ is a representation of $K$ and is equivalent to $U^{\pi_{\varphi}}$.

The proof is straightforward [2, Theorem 1] and follows from the above equation. We say that $K$ has small $H$-invariant neighborhoods ( $K \in[\mathrm{SIN}]_{H}$ ) provided there exists a neighborhood basis $\mathfrak{B}$ of $e$ in $K$ such that $t V=V t$ for all $t \in H$ and $V \in \mathfrak{B}$. As in the group case, the above 
description of induced representations in terms of positive definite measures can now be used to prove extension results on positive definite functions. In fact, the proofs of [11, Lemma 2.1 and Satz 2] carry over to our situation and show the following

Proposition 1.12. Let $K$ be a hypergroup having a Haar measure, and suppose that $H$ is a closed subgroup of $K$ such that $K \in[\mathrm{SIN}]_{H}$. Then, given any indecomposable $\gamma \in P(H)$, there exists an indecomposable $\varphi \in P(K)$ such that $\varphi \mid H=\gamma$. Consequently every irreducible representation of $H$ is equivalent to a subrepresentation of $\rho \mid H$ for some irreducible representation $\rho$ of $K$.

A stronger extension result for commutative hypergroups will be proved in $\S 2$.

2. Representations of central hypergroups. Recall that a locally compact hypergroup $K$ is called central or a $Z$-hypergroup if $K /(Z(K) \cap$ $G(K))$ is compact. It follows from [13, 7.2.A] and Lemma 1.8 that such hypergroups possess left Haar measures and are unimodular. In this section $K$ will always denote a central hypergroup unless otherwise stated and $Z$ a closed subgroup of $G(K) \cap Z(K)$ such that $K / Z$ is compact. It is easy to see then that $K=C Z$ for some compact set $C$ in $K$. Haar measures on $K, Z$ and $K / Z$ will be normalized so that $K / Z$ has measure one and Weil's formula holds. The proof of the following proposition is modelled after [8, §2, Proposition], but there are some difficulties arising from the facts that ${ }_{x}(f g) \neq_{x} f_{x} g$ in general and continuous representations of $K$ need not be unitary.

Proposition 2.1. Let $\rho$ be an irreducible representation of $K$. Then

(i) For each choice of $u, v, u^{\prime}, v^{\prime}$ in $H_{\rho}$ the function

$$
x \rightarrow\langle\rho(x) u, v\rangle \overline{\left\langle\rho(x) u^{\prime}, v^{\prime}\right\rangle}
$$

is constant on cosets of $Z$ and defines a continuous function on $K / Z$.

(ii) To $\rho$ corresponds a positive real number $c_{\rho}=\int_{K / Z}|\langle\rho(x) u, v\rangle|^{2} d \dot{x}$, where $u$ and $v$ are any vectors of norm 1 in $H_{\rho}$, and

$$
\int_{K / Z}\langle\rho(x) u, v\rangle \overline{\left\langle\rho(x) u^{\prime}, v^{\prime}\right\rangle} d \dot{x}=c_{\rho}\left\langle u, u^{\prime}\right\rangle \overline{\left\langle v, v^{\prime}\right\rangle}
$$

for all $u, v, u^{\prime}, v^{\prime}$ in $H_{\rho}$.

Proof. Since $\rho$ is irreducible and unitary on $Z$, we have $\rho(z)=\lambda(z) I_{\rho}$ for $z \in Z$, where $|\lambda(z)|=1$. Hence the continuous function in (i) is 
constant on cosets of $Z$, and (i) follows. Consider

$$
\phi\left(u, v, u^{\prime}, v^{\prime}\right)=\int_{K / Z}\langle\rho(x) u, v\rangle \overline{\left\langle\rho(x) u^{\prime}, v^{\prime}\right\rangle} d \dot{x} .
$$

For fixed $u, u^{\prime}, \phi\left(u, u^{\prime}, v, v^{\prime}\right)$ can be represented by a bounded operator. Specifically there exists a family $B_{u, u^{\prime}}$ of bounded operators on $H_{\rho}$ such that $\left\langle B_{u, u^{\prime}}\left(v^{\prime}\right), v\right\rangle=\phi\left(u, v, u^{\prime}, v^{\prime}\right)$. Let $f(x)=\langle\rho(x) u, v\rangle$ and $g(x)$ $=\overline{\left\langle\rho(x) u^{\prime}, v^{\prime}\right\rangle}$. Then clearly $f$ and $g$ satisfy the hypothesis of Lemma 1.6. Therefore

$$
\begin{aligned}
\left\langle B_{u, u^{\prime}}\left(\rho(y) v^{\prime}\right), v\right\rangle & =\int_{K / Z}\langle\rho(x) u, v\rangle \overline{\left\langle\rho(y)^{*} \rho(x) u^{\prime}, v^{\prime}\right\rangle} d \dot{x} \\
& =\int_{K / Z} f(x) g(\check{y} * x) d \dot{x}=\int_{K / Z} f(y * x) g(x) d \dot{x} \\
& =\int_{K / Z}\left\langle\rho(x) u, \rho(y)^{*} v\right\rangle \overline{\left\langle\rho(x) u^{\prime}, v^{\prime}\right\rangle} d \dot{x} \\
& =\left\langle B_{u, u^{\prime}}\left(v^{\prime}\right), \rho(y)^{*} v\right\rangle
\end{aligned}
$$

for all $y \in K$ and $u, u^{\prime}, v, v^{\prime} \in H_{\rho}$. Hence $B_{u, u^{\prime}} \rho(y)=\rho(y) B_{u, u^{\prime}}$ for all $y \in K$ and $u, u^{\prime} \in H_{\rho}$, so that $B_{u, u^{\prime}}=\beta\left(u, u^{\prime}\right) I_{\rho}, \beta\left(u, u^{\prime}\right) \in \mathbf{C}$, by Schur's Lemma. Now one easily verifies

$$
\phi\left(u, u^{\prime}, v, v^{\prime}\right)=\phi\left(v^{\prime}, v, u^{\prime}, u\right)
$$

and this implies

$$
\beta\left(u, u^{\prime}\right)\left\langle v^{\prime}, v\right\rangle=\beta\left(v^{\prime}, v\right)\left\langle u, u^{\prime}\right\rangle .
$$

It follows that $\beta(v, v)$ is constant on $\|v\|=1$. Denoting this constant by $c_{\rho}$, we obtain

$$
\int_{K / Z}\langle\rho(x) u, v\rangle \overline{\left\langle\rho(x) u^{\prime}, v^{\prime}\right\rangle} d \dot{x}=c_{\rho}\left\langle u, u^{\prime}\right\rangle \overline{\left\langle v, v^{\prime}\right\rangle} .
$$

Finally, if $\|u\|=\|v\|=1, u^{\prime}=u$ and $v^{\prime}=v$, we get

$$
c_{\rho}=\int_{K / Z}|\langle\rho(x) u, v\rangle|^{2} d \dot{x} .
$$

Recall that $P(K)$ is the set of all bounded continuous positive definite functions on an arbitrary hypergroup $K$. Moreover, let us denote by $P^{1}(K)$ the convex set of $\varphi \in P(K)$ such that $\varphi(e)=\|\varphi\|_{\infty}=1$, and by ex $P^{1}(K)$ the set of extreme points of $P^{1}(K)$. It is easily seen that $|\varphi(y * x)-\varphi(x)| \leq 2(1-\operatorname{Re} \varphi(\check{y}))$ holds for $\varphi \in P^{1}(K)$ and $x, y \in K$. Using this fact one shows as in the group case [5, (13.5.2)] that on $P^{1}(K)$ 
the weak *-topology of $L^{\infty}(K)$ coincides with the topology of uniform convergence on compact subsets of $K$. Moreover, the canonical mapping $\varphi \rightarrow \pi_{\varphi}$ from ex $P^{1}(K)$ onto $\hat{K}$ is open and continuous [5, (3.4.11)].

Returning now to $Z$-hypergroups, it is clear that $c_{\rho}$ does only depend on the equivalence class of $\rho$.

LEMMA 2.2. The function $\rho \rightarrow d_{\rho}$ on $\hat{K}$ is continuous.

Proof. Let $K=C Z$, where $C$ is compact, and let $\varphi_{0} \in \operatorname{ex} P^{1}(K)$ and $\varepsilon>0$ be given. Then by the above remarks,

$$
V=\left\{\pi_{\varphi} ; \varphi \in \operatorname{ex} P^{1}(K) \text { such that }\left|\varphi(x)-\varphi_{0}(x)\right|<\varepsilon \text { for all } x \in C\right\}
$$

is an open neighborhood of $\pi_{\varphi_{0}}$ in $\hat{K}$. Since $c_{\pi_{\varphi}}=\int_{K / Z}|\varphi(x)|^{2} d \dot{x}$, it follows that $\rho \rightarrow d_{\rho}$ is continuous.

THEOREM 2.3. If $\rho$ is an irreducible representation of $K$, then $\rho$ is finite dimensional. Moreover, the dimension function $\rho \rightarrow d_{\rho}$ is locally bounded on $\hat{K}$.

Proof. Let $\left\{e_{1}, e_{2}, \ldots, e_{n}\right\}$ be an orthonormal set in $H_{\rho}$. Then

$$
n c_{\rho}=\int_{K / Z} \sum_{i=1}^{n}\left|\left\langle\rho(x) e_{1}, e_{i}\right\rangle\right|^{2} d \dot{x} \leq \int_{K / Z}\left\|\rho(x) e_{1}\right\|^{2} d \dot{x} \leq 1
$$

by Proposition 2.1. Hence $n \leq c_{\rho}^{-1}$ and therefore $d_{\rho} \leq c_{\rho}^{-1}$. Since $\rho \rightarrow c_{\rho}$ is continuous, $\rho \rightarrow d_{\rho}$ is locally bounded.

If $\rho=\pi_{\varphi} \in \stackrel{\rho}{K}, \varphi \in \operatorname{ex} P^{1}(K)$, then $\rho(z)=\varphi(z) I_{\rho}$ for all $z \in Z$ and $\varphi \mid Z \in \hat{Z}$. We therefore have a canonical mapping $r: \hat{K} \rightarrow \hat{Z}$ defined by $\pi_{\varphi} \rightarrow \varphi \mid Z$. Clearly, $r$ is continuous.

THEOREM 2.4. Let $\rho$ and $\sigma$ be irreducible representations of $K$ such that $r(\rho)=r(\sigma)$.

(i) If $\rho$ and $\sigma$ are inequivalent, then

$$
\int_{K / Z}\langle\rho(x) u, v\rangle \overline{\left\langle\sigma(x) u^{\prime}, v^{\prime}\right\rangle} d \dot{x}=0
$$

for all $u, v \in H_{\rho}$ and $u^{\prime}, v^{\prime} \in H_{\sigma}$.

(ii) If $\left\{e_{1}, \ldots, e_{d_{\rho}}\right\}$ is an orthonormal basis of $H_{\rho}$, then

$$
\int_{K / Z}\left\langle\rho(x) e_{l}, e_{j}\right\rangle \overline{\left\langle\rho(x) e_{k}, e_{l}\right\rangle} d \dot{x}= \begin{cases}c_{\rho} & \text { if } i=k \text { and } j=l, \\ 0 & \text { otherwise. }\end{cases}
$$


Proof. (i) follows as in [8, Theorem 6.1] using Lemma 1.6 appropriately, and (ii) is a consequence of Proposition 2.1.

LEMMA 2.5. For $\lambda \in \hat{Z}, r^{-1}(\lambda)=\operatorname{supp} U^{\lambda}$.

Proof. Let $\pi=\pi_{\varphi} \in \operatorname{supp} U^{\lambda}$; then by [5, (3.4.4)] $\varphi$ can be uniformly approximated on compact subsets of $K$ by positive definite functions associated with $U^{\lambda}$. But $\left\langle U^{\lambda}(z) \xi, \xi\right\rangle=\lambda(z)\|\xi\|^{2}$ for $\xi \in H\left(U^{\lambda}\right)$ and $z \in$ $Z$, and this implies $\varphi \mid Z=\lambda$, i.e. $\pi_{\varphi} \in r^{-1}(\lambda)$. Conversely, if $\pi=\pi_{\varphi} \in$ $r^{-1}(\lambda)$, then we show that $\left\langle f^{*} * f, \varphi\right\rangle \leq \mu_{\lambda}\left(f^{*} * f\right)$, where $\mu_{\lambda}$ denotes the positive definite measure on $K$ defined by $\lambda$. Choose $h \in C_{00}^{+}(K)$ such that $T_{Z} h=1$. Then easy computations show

$$
\begin{aligned}
\left\langle f^{*} * f, \varphi\right\rangle & =\int_{Z} \int_{Z}\left(\int_{K} \int_{K} \overline{f(y)} f(x) \varphi(\check{y} * x) h(x \check{t}) h(y \check{s}) d x d y\right) d s d t \\
& =\int_{K} \int_{K} h(x) h(y) \varphi(\check{y} * x)\left|\int_{Z} f(t) \lambda(t) d t\right|^{2} d x d y \\
& \leq\left(\int_{K} h(x)\left|\varepsilon\left({ }_{x} f, 1\right)\right|^{2} d x\right)^{2}=\int_{K / Z}\left|\varepsilon\left({ }_{x} f, 1\right)\right|^{2} d \dot{x} \\
& =\mu_{\lambda}\left(f^{*} * f\right)
\end{aligned}
$$

(see the discussion before Lemma 1.11). Thus the positive linear functional $f \rightarrow\langle f, \varphi\rangle$ is majorized by the positive linear functional $f \rightarrow \mu_{\lambda}(f)$. By [5, (2.5.1)] and Lemma 1.11, given $\eta \in H_{\pi}$, there exist an operator $T$ on $H_{\pi}$ and $\xi \in H_{\pi}$ such that

$$
\left\langle\pi_{\varphi}(f) \eta, \eta\right\rangle=\left\langle U^{\lambda}(f) T \xi, T \xi\right\rangle
$$

for all $f \in C_{00}(K)$ and hence for all $f \in C^{*}(K)$. This shows that kernel $U^{\lambda} \subseteq$ kernel $\pi_{\varphi}$, i.e. $\pi_{\varphi} \in \operatorname{supp} U^{\lambda}$.

We should mention that the proof of the following theorem does not require any structure theory and is much simpler than the one given in the group case in [10, Theorem (1.4)].

THEOREM 2.6. The canonical map $r: \hat{K} \rightarrow \hat{Z}$ is a local homeomorphism. In particular, $r$ is an open map and $r^{-1}(\lambda)$ is discrete for all $\lambda \in \hat{Z}$.

Proof. We first show that $r$ is locally injective and $r^{-1}(\lambda)$ is discrete for every $\lambda \in \hat{Z}$. Let $\pi_{0}=\pi_{\varphi_{0}} \in \hat{K}, \varphi_{0} \in \operatorname{ex} P^{1}(K)$ and assume $r$ is not injective on every neighborhood of $\pi_{0}$ in $\hat{K}$. By the earlier discussion and $[5,3.4 .11]$, the mapping $\varphi \rightarrow \pi_{\varphi}$ as a mapping from ex $P^{1}(K)$ onto $\hat{K}$ is 
open. Let $K=C Z, C$ compact and $V_{n}=\left\{\pi_{\varphi} ; \varphi \in \operatorname{ex} P^{1}(K), \mid \varphi(x)-\right.$ $\varphi_{0}(x) \mid<1 / n$ for all $\left.x \in C\right\}$. Then $V_{n}$ is an open neighborhood of $\pi_{0}$, and there exist $\pi_{n}=\pi_{\varphi_{n}}$ and $\rho_{n}=\pi_{\psi_{n}}$ in $V_{n}$ such that $r\left(\pi_{n}\right)=r\left(\rho_{n}\right)$ but $\pi_{n} \neq \rho_{n}$. Let $\varphi_{n}(x)=\left\langle\pi_{n}(x) \xi_{n}, \xi_{n}\right\rangle(n \geq 0)$ and $\psi_{n}(x)=\left\langle\rho_{n}(x) \eta_{n}, \eta_{n}\right\rangle(n \geq 1)$ and set $\delta=\int_{K / Z}\left|\varphi_{0}(x)\right|^{2} d \dot{x}>0$. Then for sufficiently large $n$,

$$
\begin{aligned}
\frac{\delta}{2} & \leq \int_{K / Z}\left|\varphi_{n}(x)\right|^{2} d \dot{x}=\int_{K / Z}\left|\left\langle\pi_{n}(x) \xi_{n}, \xi_{n}\right\rangle\right|^{2} d \dot{x} \\
& =\int_{K / Z}\left(\left\langle\pi_{n}(x) \xi_{n}, \xi_{n}\right\rangle-\left\langle\rho_{n}(x) \eta_{n}, \eta_{n}\right\rangle\right)\left\langle\pi_{n}(x) \xi_{n}, \xi_{n}\right\rangle d \dot{x}
\end{aligned}
$$

by Theorem 2.4. Now if $x=c z, c \in C, z \in Z$, then the integrand is $\leq\left|\varphi_{n}(c)-\psi_{n}(c)\right| \leq 2 / n$. This is a contradiction.

It remains to show that $r$ is open. For this notice that if $E \subseteq \hat{Z}$ and $\lambda \in \bar{E}$, then $\operatorname{ker} U^{\lambda} \supseteq \cap_{\tau \in E} \operatorname{ker} U^{\tau}$. In fact, if for $f \in C_{00}(K)$ we define $\varepsilon_{\lambda}(f) \in H\left(U^{\lambda}\right)$ by

$$
\varepsilon_{\lambda}(f)(y)=\varepsilon(f, 1)(y)=\int_{Z} f(y z) \lambda(z) d z,
$$

then the set of all $\varepsilon_{\lambda}(f), f \in C_{00}(K)$, is total in $H\left(U^{\lambda}\right)$, and we have

$$
\begin{aligned}
& \left\langle U^{\lambda}(\varphi) \varepsilon_{\lambda}(f), \varepsilon_{\lambda}(g)\right\rangle \\
& \quad=\int_{K} \varphi(x) \int_{K / Z}\left(\int_{Z} f(\check{x} * y z) \lambda(z) d z \cdot \int_{Z} \overline{g(y z)} \overline{\lambda(z)} d z\right) d \dot{y} d x
\end{aligned}
$$

for $\varphi, f, g \in C_{00}(K)$. Hence $\left\langle U^{\lambda}(\varphi) \varepsilon_{\lambda}(f), \varepsilon_{\lambda}(g)\right\rangle$ is the limit of the $\left\langle U^{\tau}(\varphi) \varepsilon_{\tau}(f), \varepsilon_{\tau}(g)\right\rangle, \tau \in E$, and the same holds for $a \in C^{*}(K)$ instead of $\varphi \in C_{00}(K)$ by continuity and density arguments.

Let now $V \subseteq \hat{K}$ be open and set $A=r^{-1}(\hat{Z} \backslash r(V))$. Then $A$ is closed. In fact, if $\pi_{0} \in \bar{A}$, then $r\left(\pi_{0}\right) \in r(\bar{A}) \subseteq \overline{r(A)}=\overline{(\hat{Z} \backslash r(V))}$, hence

$$
\operatorname{ker} U^{r\left(\pi_{0}\right)} \supseteq \bigcap\left\{\operatorname{ker} U^{\tau} ; \tau \in \hat{Z} \backslash r(V)\right\}=\bigcap_{\pi \in A} \operatorname{ker} \pi,
$$

i.e. $r^{-1}\left(r\left(\pi_{0}\right)\right) \subseteq A \subseteq \hat{K} \backslash V$ and $\pi_{0} \in A$. But $A=r^{-1}(r(A))$ and $A$ closed implies $r(A)$ is closed by the same arguments.

Proposition 2.7. $H\left(U^{\lambda}\right)$ is a Hilbert algebra for every $\lambda \in \hat{Z}$.

Proof. $H\left(U^{\lambda}\right)$ consists of all Bourbaki measurable functions $f: K \rightarrow \mathbf{C}$ such that $\dot{x} \rightarrow|f(x)|^{2}$ is integrable and $f(x t)=\overline{\lambda(t)} f(x)$ for $x \in K, t \in Z$. Set $H=H\left(U^{\lambda}\right)$, and let $A$ denote the subspace of all continuous functions 
in $H$. For $f \in A$ and $g \in H$ we define $g^{*} \in H$ and $f \circ g \in A$ by $g^{*}(x)$ $=\overline{g(\check{x})}$ and

$$
f \circ g(x)=\left\langle U^{\lambda}(\check{x}) f, g^{*}\right\rangle=\int_{K / Z} f(x * y) g(\check{y}) d \dot{y} .
$$

Then $\|f \circ g\| \leq\|f\| \cdot\|g\|$, and $A$ becomes a Hilbert algebra. Put $\tilde{\varphi}=\varepsilon(\varphi, 1)$ for $\varphi \in C_{00}(K)$; then for $g \in A$ we have

$$
\begin{aligned}
\tilde{\varphi} \circ g(x) & =\int_{K / Z} \tilde{\varphi}(x * y) g(\check{y}) d \dot{y}=\int_{K / Z} \int_{Z} \varphi(x * y t) \lambda(t) g(\check{y}) d t d \dot{y} \\
& =\int_{K / Z} \int_{Z} \varphi(x * y t) g((y t)) d t d \dot{y}=\int_{K} \varphi(x * y) g(\check{y}) d y \\
& =\int_{K} \varphi(y) g(\check{y} * x) d y=U^{\lambda}(\varphi) g(x) .
\end{aligned}
$$

Choose $\varphi_{n} \in C_{00}(K), \quad n \in \mathbf{N}$, such that $U^{\lambda}\left(\varphi_{n}\right) g \rightarrow g$, i.e. $g=$ $\lim _{n \rightarrow \infty} \tilde{\varphi}_{n} \circ g \in \overline{A \circ A}$. Hence $A \circ A$ is dense in $H$. Now $g \rightarrow \tilde{\varphi} \circ g$ and $U^{\lambda}(\varphi)$ are continuous mappings in $H$, so we obtain $\tilde{\varphi} \circ g=U^{\lambda}(\varphi) g$ for all $g \in H$ and $\varphi \in C_{00}(K)$. Finally, by Lemma 1.6, we have $f \circ g(x)=$ $\int_{K / Z} f(y) g(\check{y} * x) d \dot{y}$ for all $f, g \in A$. Thus $f \rightarrow f \circ g$ can be extended to $H$ by setting $f \circ g(x)=\int_{K / Z} f(y) g(\check{y} * x) d \dot{y}$ for $f \in H$ and $g \in A$. Again, $\|f \circ g\| \leq\|f\| \cdot\|g\|$, hence every $f \in H$ is bounded, and $H$ is the maximal Hilbert algebra.

For every $\rho \in r^{-1}(\lambda)=\operatorname{supp} U^{\lambda}$ we select an orthonormal basis $\left\{e_{1}, \ldots, e_{d_{\rho}}\right\}$ of $H_{\rho}$ and define the coordinate functions $\rho_{i j}(x)=$ $\left\langle\rho(\check{x}) e_{\imath}, e_{\rho}\right\rangle, 1 \leq i, j \leq d_{\rho}$. Then $\rho_{l j} \in A$, the subspace of all continuous functions in $H\left(U^{\lambda}\right)$. If $k_{\rho}=c_{\rho}^{-1}$, then

$$
B=\left\{k_{\rho}^{1 / 2} \rho_{i j} ; \rho \in r^{-1}(\lambda), 1 \leq i, j \leq d_{\rho}\right\}
$$

is an orthonormal system in $H\left(U^{\lambda}\right)$ (Theorem 2.4). Let $L_{\rho}^{j}$ denote the linear span of $\left\{\rho_{i j} ; 1 \leq i \leq d_{\rho}\right\}, 1 \leq j \leq d_{\rho}$.

Proposition 2.8. $B$ is an orthonormal basis for $H\left(U^{\lambda}\right)$, i.e. $H\left(U^{\lambda}\right)=$ $\bigoplus\left\{L_{\rho}^{j} ; \quad \rho \in r^{-1}(\lambda), \quad 1 \leq j \leq d_{\rho}\right\} . U^{\lambda}$ is equivalent to the direct sum $\bigoplus_{\rho \in r^{-1}(\lambda)} d_{\rho} \cdot \rho$.

Proof. First we observe that

$$
\rho_{l j}(x * y)=\sum_{k=1}^{d_{\rho}} \rho_{l k}(x) \rho_{k J}(y) .
$$


Moreover, using Proposition 2.1 and denoting by $\circ$ the product in the Hilbert algebra $H\left(U^{\lambda}\right)$ (see the proof of Proposition 2.7), we obtain

$$
\rho_{i j}^{\circ} \rho_{k l}(x)=c_{\rho} \delta_{j k} \rho_{i l}(x) .
$$

Define now $J_{\rho}$ to be the linear span of $\left\{\rho_{i j} ; 1 \leq i, j \leq d_{\rho}\right\}$. Then $J_{\rho}$ is a right ideal in $H\left(U^{\lambda}\right)$. In fact, for $f \in H\left(U^{\lambda}\right)$ and $x \in K$,

$$
\begin{aligned}
\rho_{i j} \circ f(x) & =\int_{K / Z} \rho_{i j}(x * y) f(\check{y}) d \dot{y} \\
& =\int_{K / Z} f(\check{y}) \sum_{k} \rho_{i k}(x) \rho_{k j}(y) d \dot{y} \\
& =\sum_{k}\left(\int_{K / Z} f(y) \rho_{k j}(\check{y}) d \dot{y}\right) \rho_{i k}(x) .
\end{aligned}
$$

Next we claim that $L_{\rho}^{j}$ is $U^{\lambda}$-invariant and $U^{\lambda} \mid L_{\rho}^{j}$ is equivalent to $\rho$. The equality

$$
U^{\lambda}(x) \rho_{i j}(y)=\rho_{i j}(\check{x} * y)=\sum_{l} \rho_{i l}(\check{x}) \rho_{l j}(y)
$$

shows that $U^{\lambda}(x) \rho_{i j} \in L_{\rho}^{j}$. For the isomorphism $\phi: L_{\rho}^{j} \rightarrow H_{\rho}$ defined by $\phi\left(k_{\rho}^{1 / 2} \rho_{i j}\right)=e_{l}$, we have

$$
\begin{aligned}
\phi U^{\lambda}(x)\left(k_{\rho}^{1 / 2} \rho_{i j}\right) & =\sum_{l} \rho_{i l}(\check{x}) \phi\left(k_{\rho}^{1 / 2} \rho_{l j}\right) \\
& =\sum_{l}\left\langle\rho(x) e_{l}, e_{l}\right\rangle e_{l}=\rho(x) e_{i} ;
\end{aligned}
$$

hence $\phi U^{\lambda}(x)=\rho(x) \phi$. Finally, let $E$ denote the orthogonal complement of $\oplus\left\{L_{\rho}^{j} ; \rho \in r^{-1}(\lambda), 1 \leq j \leq d_{\rho}\right\}$ and assume $E \neq 0$. Then, since supp $U^{\lambda}$ is discrete (Theorem 2.6), there exists an irreducible subspace $F$ of $E$ (see [20, Theorem 1.7]). For a suitable $\rho \in r^{-1}(\lambda), U^{\lambda} \mid F$ is equivalent to $\rho$, hence to $U^{\lambda} / L_{\rho}^{1}$. Let $\phi$ be an intertwining operator and put

$$
u_{i}=\phi\left(k_{\rho}^{1 / 2} \rho_{i 1}\right) \in F .
$$

For $\varphi \in C_{00}(K)$ we have

$$
\tilde{\varphi} \circ u_{i}=U^{\lambda}(\varphi) u_{i}=\phi\left(\left(U^{\lambda}(\varphi) k_{\rho}^{1 / 2} \rho_{i 1}\right)\right)=\phi\left(\tilde{\varphi} \circ k_{\rho}^{1 / 2} \rho_{i 1}\right) .
$$

On the other hand,

$$
\phi\left(\rho_{i i} \circ \rho_{i 1}\right)=\phi\left(c_{\rho} \rho_{i 1}\right)=c_{\rho} u_{i} .
$$


Choosing now $\varphi_{n} \in C_{00}(K)$ such that $\rho_{u l}=\lim \tilde{\varphi}_{n}$, it follows that

$$
\begin{aligned}
u_{i} & =k_{\rho} \phi\left(\rho_{i i} \circ \rho_{t 1}\right)=k_{\rho} \lim \phi\left(\tilde{\varphi}_{n} \circ \rho_{i 1}\right) \\
& =k_{\rho}^{1 / 2} \lim \tilde{\varphi}_{n} \circ u_{i}=k_{\rho}^{1 / 2} \rho_{u l} \circ u_{l} \in J_{\rho},
\end{aligned}
$$

since $J_{\rho}$ is a right ideal. Thus $u_{i} \in F \cap J_{\rho}=0$, a contradiction. Hence $H\left(U^{\lambda}\right)=\oplus\left\{L_{\rho}^{\jmath} ; \rho \in r^{-1}(\lambda), 1 \leq j \leq d_{\rho}\right\}$, and this implies immediately the assertion on $U^{\lambda}$.

It should be mentioned that the following theorem is proved in a similar way as the character formula in [19, Theorem 2.15].

THEOREM 2.9. A continuous function $h$ on $K$ satisfies

$$
h(y) h(z)=\int_{K / Z} h(x * y * \check{x} * z) d \dot{x} \quad \text { and } \quad h(e) \neq 0
$$

if and only if $h(y)=c_{\rho} \operatorname{tr} \rho(y)$ for some $\rho \in \hat{K}$.

Proof. Applying Proposition 2.1, one shows as in the case of a compact hypergroup that every function $c_{\rho} \operatorname{tr} \rho, \rho \in \hat{K}$, satisfies the above formula. Conversely, suppose $h(e) \neq 0$ and the given equation holds for $h$. Then

$$
h(y) h(t)=\int_{K / Z} h(x * y t * \check{x}) d \dot{x}=h(y t) h(e)
$$

for $y \in K, t \in Z$. In particular, $\lambda(t)=\overline{h(e)^{-1} h(t)}$ defines a character on $Z$. Since $h(y t)=\lambda(\tilde{t}) h(y)$ for $y \in K$ and $t \in Z$, for every $\rho \in \operatorname{supp} U^{\lambda}$ we can define a bounded operator $T_{\rho}$ on $H_{\rho}$ by

$$
\left\langle T_{\rho} \xi, \eta\right\rangle=\int_{K / Z} h(x)\langle\rho(x) \xi, \eta\rangle d \dot{x}, \quad \xi, \eta \in H_{\rho}
$$

As $0 \neq h \in H\left(U^{\lambda}\right)$, there exists a $\rho$ such that $T_{\rho} \neq 0$. Now, by Lemma 1.6

$$
\begin{aligned}
\left\langle h(e) T_{\rho} \xi, \eta\right\rangle & =\int_{K / Z} \int_{K / Z} h(\check{x} * y * x)\langle\rho(y) \xi, \eta\rangle d \dot{x} d \dot{y} \\
& =\int_{K / Z} \int_{K / Z} h(y * x)\langle\rho(x * y) \xi, \eta\rangle d \dot{x} d \dot{y}
\end{aligned}
$$


and again using Lemma 1.6 repeatedly, it follows that

$$
\begin{aligned}
\left\langle\rho(a) h(e) T_{\rho} \xi, \eta\right\rangle & =\int_{K / Z} \int_{K / Z} h(y * x)\langle\rho(a * x) \rho(y) \xi, \eta\rangle d \dot{x} d \dot{y} \\
& =\int_{K / Z} \int_{K / Z} h(y * a * x)\langle\rho(x) \rho(y) \xi, \eta\rangle d \dot{y} d \dot{x} \\
& =\int_{K / Z} \int_{K / Z} h(\check{x} * y * x)\langle\rho(y) \rho(a) \xi, \eta\rangle d \dot{x} d \dot{y} \\
& =\left\langle h(e) T_{\rho} \rho(a) \xi, \eta\right\rangle .
\end{aligned}
$$

Therefore $T_{\rho}$ is a scalar operator, $T_{\rho}=\alpha \cdot I_{\rho}$. Let $\left\{e_{1}, \ldots, e_{d_{\rho}}\right\}$ be an orthonormal basis in $H_{\rho}$. Then for $z \in K$,

$$
\begin{aligned}
\alpha h(z) d_{\rho} & =h(z) \sum_{i=1}^{d_{\rho}} \int_{K / Z} h(y)\left\langle\rho(y) e_{i}, e_{i}\right\rangle d \dot{y} \\
& =\int_{K / Z} \int_{K / Z} h(\check{x} * y * x * z) \operatorname{tr} \rho(y) d \dot{x} d \dot{y} \\
& =\int_{K / Z} \int_{K / Z} h(y * z) \operatorname{tr} \rho(x * y * \check{x}) d \dot{x} d \dot{y} \\
& =c_{\rho} d_{\rho} \int_{K / Z} h(y * z) \operatorname{tr} \rho(y) d \dot{y} \\
& =c_{\rho} d_{\rho} \sum_{i=1}^{d_{\rho}} \int_{K / Z} h(y)\left\langle\rho(y) \rho(\check{z}) e_{l}, e_{l}\right\rangle d \dot{y} \\
& =c_{\rho} d_{\rho} \alpha \operatorname{tr} \rho(\check{z}) .
\end{aligned}
$$

Hence $h(z)=c_{\rho} \operatorname{tr} \bar{\rho}(z)$, where $\bar{\rho}$ is the conjugate representation of $\rho$.

REMARK 2.10. If $K$ is a commutative central hypergroup, then using Lemma 1.6 and Theorem 2.4, it can easily be shown that every bounded multiplicative continuous function $\chi: K \rightarrow \mathbf{C}$ is hermitian, i.e. satisfies $\chi(\check{x})=\overline{\chi(x)}$ for all $x \in K($ see [4]).

We conclude this section with a generalization of [16, Corollary 2.3 and Proposition 3.6].

THEOREM 2.11. Let $K$ be a commutative hypergroup and $H$ a subhypergroup of $K$ such that $H / H \cap G(K)$ is compact. Then every character of $H$ extends to a character of $K$. 
Proof. Let $\chi \in \hat{H}, F=H \cap G(K)$ and $\lambda=\chi \mid F$. We first claim that if $\alpha \in \operatorname{supp}_{K} U^{\lambda}$, then $\alpha \mid F=\lambda$. Assuming that $\alpha \mid F \neq \lambda$, by the regularity of $L^{1}(F)$ there exists an $f \in L^{1}(F)$ such that $\hat{f}(\bar{\alpha} \mid \bar{F}) \neq 0$ and $\hat{f}(\bar{\lambda})=0$. It follows that ${ }_{K} U^{\lambda}\left(g * \mu_{f}\right)=0$ for all $g \in C_{00}(K)$ since

$$
\left(U^{\lambda}\left(g * \mu_{f}\right) \xi\right)(x)=\hat{f}(\bar{\lambda}) \int_{K} \widehat{y} \widehat{g \mid F}(\bar{\lambda}) \xi(\check{y} * x) d y .
$$

Hence

$$
0=\widehat{g * \mu_{f}}(\bar{\alpha})=\hat{g}(\bar{\alpha}) \hat{f}(\overline{\alpha \mid F}) \quad \text { for all } g \in C_{00}(K),
$$

which contradicts $\hat{f}(\overline{\alpha \mid F}) \neq 0$.

We show next that if $h \in C_{00}(H)$ such that ${ }_{K} U^{\lambda}\left(g * \mu_{h}\right)=0$ for all $g \in C_{00}(K)$, then ${ }_{H} U^{\lambda}(h)=0$. It clearly suffices to show that $\int_{F} \lambda(t)(\psi * h)(t) d t=0$ for all $\psi \in C_{00}(H)$. But this follows easily from the assumption, since

$$
0={ }_{K} U^{\lambda}\left(g * \mu_{h}\right) \xi(\varphi, 1)(e)=\int_{F} \lambda(t)[(\varphi * g) \mid H * h](t) d t
$$

for all $\varphi, g \in C_{00}(K)$.

Let now $\Delta=\{\alpha \in \hat{K} ; \alpha \mid F=\lambda\}$; then by what we have verified above

$$
\left\{f \in L^{1}(K) ; \hat{f}(\bar{\alpha})=0 \text { for all } \alpha \in \Delta\right\} \subseteq\left\{f \in L^{1}(K) ; U^{\lambda}(f)=0\right\} .
$$

Assume $\chi \notin \Delta \mid H$ and choose $f \in C_{00}^{+}(H)$ such that $T_{F} f=1$ on $H / F$ and $f(e)>0$. Then by the orthogonality relations

$$
\begin{aligned}
0 & =\int_{H / F} \alpha(x) \overline{\chi(x)} d \dot{x}=\int_{H / F} \int_{F} \alpha(x t) \overline{\chi(x t)} f(x t) d t d \dot{x} \\
& =\int_{H} \alpha(y) \overline{\chi(y)} f(y) d y=\widehat{f \cdot \bar{\chi}}(\overline{\alpha \mid H})
\end{aligned}
$$

for all $\alpha \in \Delta$. Thus

$$
\left(g * \mu_{f \cdot \bar{\chi}}\right)^{\wedge}(\bar{\alpha})=\hat{g}(\bar{\alpha}) \widehat{f \cdot \bar{\chi}}(\overline{\alpha \mid H})=0
$$

for $\alpha \in \Delta$ and $g \in C_{00}(K)$, and therefore

$$
{ }_{K} U^{\lambda}\left(g * \mu_{f \cdot \bar{x}}\right)=0 \quad \text { for all } g \in C_{00}(K) \text {. }
$$

This shows ${ }_{H} U^{\lambda}(f \cdot \bar{\chi})=0$. But $\chi \in \operatorname{supp}_{H} U^{\lambda}$ (Lemma 2.5), so

$$
\int_{H} f(x)|\chi(x)|^{2} d x=0
$$

a contradiction. 
3. Plancherel theorem and Inversion formula. Our proof of the Plancherel theorem follows the lines of [9, Theorem 4.1]. But there seem to be some gaps in the proof given in [9], one concerning the application of Fubini's theorem on page 435 . To be very careful, we include the following

Lemma 3.1. For $f \in L^{1}(K)$ and $\lambda \in \hat{Z}$ define $\varepsilon_{\lambda}(f)$ on $K$ by

$$
\varepsilon_{\lambda}(f)(x)= \begin{cases}\widehat{f f \mid Z}(\bar{\lambda}) & \text { if }{ }_{x} f \mid Z \in L^{1}(Z), \\ 0 & \text { otherwise. }\end{cases}
$$

Then the function $(x, \lambda) \rightarrow \varepsilon_{\lambda}(f)(x)$ on $K \times \hat{Z}$ is measurable.

Proof. Notice first that for $f \in C_{00}(K), \varepsilon_{\lambda}(f)=\varepsilon(f, 1) \in H\left(U^{\lambda}\right)$ in the notation of $\S 1$. Let $f \in L^{1}(K), u \in C_{00}^{+}(K)$ and $g=f * u$. Then ${ }_{x} g \mid Z \in L^{1}(Z)$ for all $x \in K$, and the mapping $x \rightarrow{ }_{x} g \mid Z$ from $K$ into $L^{1}(Z)$ is continuous and bounded by $\|f\|_{1}\left\|T_{H} u\right\|_{\infty}[14$, Ch. 7, §3.3]. Hence $(x, \lambda) \rightarrow{ }_{x} g \mid Z(\bar{\lambda})=\varepsilon_{\lambda}(g)(x)$ is continuous on $K \times \hat{Z}$.

Now $f$ can be approximated by a sequence $f_{n}=f * u_{n}, u_{n} \in C_{00}^{+}(K)$. Thus, following [14, Ch. 3, §4.7] one shows the existence of a null set $A_{f}$ in $K / Z$ such that for every $x \notin p^{-1}\left(A_{f}\right)$ the integrals $\int_{Z}\left|f(x t)-f_{n}(x t)\right| d t$ tend to zero. Hence ${ }_{x}\left(f_{n}\right) \mid Z$ converges to ${ }_{x} f \mid Z$ in $L^{1}(Z)$ for $x \notin p^{-1}\left(A_{f}\right)$, and $p^{-1}\left(A_{f}\right)$ is locally null by Weil's formula. Thus $p^{-1}\left(A_{f}\right) \times \hat{Z}$ is locally null in $K \times \hat{Z}$, and on the complement we have $\varepsilon_{\lambda}(f)(x)=$ $\lim _{n \rightarrow \infty} \varepsilon_{\lambda}\left(f_{n}\right)(x)$. By [3, Ch. IV, §4, Theorem 2] $(x, \lambda) \rightarrow \varepsilon_{\lambda}(f)(x)$ is measurable.

THEOREM 3.2. (Plancherel formula). For $f, g \in L^{1}(K) \cap L^{2}(K)$ the function $\lambda \rightarrow \sum_{\rho \in r^{-1}(\lambda)} k_{\rho} \operatorname{tr}\left(\rho(f) \rho(g)^{*}\right)$ is in $L^{1}(\hat{Z})$ and satisfies

$$
\int_{K} f(x) \overline{g(x)} d x=\int_{\hat{Z}_{\rho \in r^{-1}(\lambda)}} k_{\rho} \operatorname{tr}\left(\rho(f) \rho(g)^{*}\right) d \lambda .
$$

Proof. For $f, g \in L^{1}(K) \cap L^{2}(K)$ we have ${ }_{x} f\left|Z,{ }_{x} g\right| Z \in L^{1}(Z) \cap$ $L^{2}(Z)$ outside $p^{-1}(A)$ for some set $A$ of measure zero in $K / Z$. So we can apply the Plancherel formula for abelian locally compact groups to the functions ${ }_{x} f \mid Z$ and ${ }_{x} g \mid Z$ :

$$
\begin{aligned}
\langle f, g\rangle & =\int_{K / Z} \int_{Z} f(t)_{x} \overline{g(t)} d t d \dot{x} \\
& =\int_{K / Z} \int_{\dot{Z}} \varepsilon_{\lambda}(f)(x) \overline{\varepsilon_{\lambda}(g)(x)} d \lambda d \dot{x}
\end{aligned}
$$


Putting first $f=g$ one can see that $(\dot{x}, \lambda) \rightarrow \varepsilon_{\lambda}(f)(x) \overline{\varepsilon_{\lambda}(g)(x)}$ is in $L^{1}(K / Z \times \hat{Z}, d \dot{x} \times d \lambda)$, and Fubini's theorem applies:

(i) $\dot{x} \rightarrow \varepsilon_{\lambda}(f)(x) \overline{\varepsilon_{\lambda}(g)(x)}$ is in $L^{1}(K / Z)$ for almost every $\lambda \in \hat{Z}$;

(ii) $\lambda \rightarrow \int_{K / Z} \varepsilon_{\lambda}(f)(x) \overline{\varepsilon_{\lambda}(g)(x)} d \dot{x}$ is in $L^{1}(\hat{Z})$; and

(iii) $\langle f, g\rangle=\int_{\hat{Z}} \int_{K / Z} \varepsilon_{\lambda}(f)(x) \varepsilon_{\lambda}(g)(x) d \dot{x} d \lambda$.

From (i) it follows that $\varepsilon_{\lambda}(f) \in H\left(U^{\lambda}\right)$ for almost all $\lambda \in \hat{Z}$. Let $\lambda \in \hat{Z}$ be such that $\varepsilon_{\lambda}(f), \varepsilon_{\lambda}(g) \in H\left(U^{\lambda}\right)$. Then for the basis $\left\{k^{1 / 2} \rho_{i j} ; \rho \in r^{-1}(\lambda), 1 \leq i, j \leq d_{\rho}\right\}$ of $H\left(U^{\lambda}\right)$ we have

$$
\begin{aligned}
\left\langle\varepsilon_{\lambda}(f), \rho_{i j}\right\rangle & =\int_{K / Z} \int_{Z} f(x t) \lambda(t)\left\langle\rho(x) e_{j}, e_{i}\right\rangle d t d \dot{x} \\
& =\int_{K / Z} \int_{Z} f(x t)\left\langle\rho(x t) e_{j}, e_{i}\right\rangle d t d \dot{x}=\left\langle\rho(f) e_{j}, e_{i}\right\rangle
\end{aligned}
$$

Thus the following holds:

$$
\begin{aligned}
\int_{K / Z} \varepsilon_{\lambda}(f)(x) \overline{\varepsilon_{\lambda}(g)(x)} & \dot{x}=\left\langle\varepsilon_{\lambda}(f), \varepsilon_{\lambda}(g)\right\rangle \\
& =\sum_{\rho \in r^{-1}(\lambda)} \sum_{i, j=1}^{d_{\rho}}\left\langle\varepsilon_{\lambda}(f), k_{\rho}^{1 / 2} \rho_{i j}\right\rangle\left\langle k_{\rho}^{1 / 2} \rho_{i j}, \varepsilon_{\lambda}(g)\right\rangle \\
& =\sum_{\rho \in r^{-1}(\lambda)} \sum_{i, j=1}^{d_{\rho}} k_{\rho}\left\langle\rho(f) e_{j}, e_{i}\right\rangle\left\langle e_{i}, \rho(g) e_{j}\right\rangle \\
& =\sum_{\rho \in r^{-1}(\lambda)} k_{\rho} \operatorname{tr}\left(\rho(f) \rho(g)^{*}\right) .
\end{aligned}
$$

Now both assertions follow by means of (ii) and (iii).

We didn't succeed in proving that a Borel measure $\mu$ on $\hat{K}$ can be defined by

$$
\mu(E)=\int_{\hat{Z}}\left|E \cap r^{-1}(\lambda)\right| d \lambda .
$$

Even in the case of a $Z$-group it is not clear at all whether $\lambda \rightarrow\left|E \cap r^{-1}(\lambda)\right|$ is measurable.

We are going to prove now the Inversion formula for linear combinations of functions in $L^{1}(K) \cap P(K)$. We would like to mention at this point that if $K$ is a hypergroup such that $K / G(K)$ is compact, then a continuous positive definite function is easily seen to be bounded. In particular, this holds for $Z$-hypergroups.

LEMMA 3.3. If $f \in L^{1}(K) \cap P(K)$, then $\rho(f)$ is a positive operator for all $\rho \in \hat{K}$. 
Proof. Let $\lambda$ denote the left regular representation of $L^{1}(K)$ in $L^{2}(K)$ as well as its extension to $C^{*}(K)$. We have

$$
\langle\lambda(f) g, g\rangle=\int_{K} \int_{K} \overline{g(x)} g(y) f(x * \check{y}) d x d y \geq 0
$$

for all $g \in C_{00}(K)$. Hence $\lambda(f) \geq 0$ and $\lambda(f)=T^{*} T$ for some $T \in$ $\lambda\left(C^{*}(K)\right)$. Suppose we have already shown that $\lambda$ is faithful on $C^{*}(K)$. Then for $\rho \in \hat{K}$,

$$
\rho(f)=\rho\left(\lambda^{-1}\left(T^{*} T\right)\right)=\rho\left(\lambda^{-1}(T)\right) * \rho\left(\lambda^{-1}(T)\right) \geq 0 .
$$

Let $Z=Z(K) \cap G(K), r: \hat{K} \rightarrow \hat{Z}$ and $\varphi=r(\rho)$. Since $\rho$ is a subrepresentation of $U^{\varphi}$, it suffices to show that kernel $\lambda \subseteq \operatorname{kernel} U^{\varphi}$, i.e. $U^{\varphi}$ is weakly contained in $\lambda$ in the sense of $[5, \S 3.4]$. To this end, as in the group case, one has to prove that every positive definite function $x \rightarrow$ $\left\langle U^{\varphi}(x) \xi, \xi\right\rangle, \xi \in H\left(U^{\varphi}\right)$, can be uniformly approximated on compact subsets of $K$ by sums of positive definite functions associated with $\lambda$. Of course, $\xi$ can be assumed to be $\varepsilon(g, 1)$ for some $g \in C_{00}(K)$. Choosing $h \in C_{00}^{+}(K)$ such that $T_{Z} h=1$, we have

$$
\begin{aligned}
& \left\langle U^{\varphi}(x) \varepsilon(g, 1), \varepsilon(g, 1)\right\rangle \\
& \quad=\int_{K} h(y)\left(\int_{Z} g(\check{x} y t) \lambda(t) d t\right)\left(\int_{Z} \overline{g(y s)} \overline{\lambda(s)} d s\right) d y .
\end{aligned}
$$

Now, given a compact subset $C$ of $K$, there exist compact sets $D$ in $K$ and $E$ in $Z$ such that

$$
\begin{aligned}
& \left\langle U^{\varphi}(x) \varepsilon(g, 1), \varepsilon(g, 1)\right\rangle \\
& \quad=\int_{D} h(y)\left(\int_{E} g(\check{x} y t) \lambda(t) d t\right)\left(\int_{E} \overline{g(y s) \lambda(s)} d s\right) d y .
\end{aligned}
$$

Choosing suitable disjoint coverings $D=\cup_{j=1}^{m} V_{j}, E=\cup_{l=1}^{r} W_{l}$ and $y_{j} \in$ $V_{J}, t_{l} \in W_{l}$ and $\xi_{J} \in L^{2}(K)$ by

$$
\xi_{j}=\sum_{l=1}^{r}\left|W_{l}\right| \lambda\left(t_{l}\right) \chi_{v_{j}} g_{t_{l}},
$$

where $\left|W_{l}\right|$ denotes the Haar measure of $W_{l}$ and $\chi_{v_{j}}$ the characteristic function of $V_{j}$, we conclude that $\left\langle U^{\varphi}(x) \varepsilon(g, 1), \varepsilon(g, 1)\right\rangle$ can be uniformly approximated on $C$ by $\sum_{j=1}^{m} h\left(y_{j}\right)\left\langle\lambda(x) \xi_{j}, \xi_{j}\right\rangle$.

THEOREM 3.4. (Inversion formula). For $f \in\left[L^{1}(K) \cap P(K)\right]$ (the linear span of $\left.L^{1}(K) \cap P(K)\right)$ and $x \in K$ the function

$$
\lambda \rightarrow \sum_{\rho \in r^{-1}(\lambda)} k_{\rho} \operatorname{tr}\left(\rho(f) \rho(x)^{*}\right)
$$


belongs to $L^{1}(\hat{Z})$ and satisfies

$$
f(x)=\int_{\hat{Z}} \sum_{\rho \in r^{-1}(\lambda)} k_{\rho} \operatorname{tr}\left(\rho(f) \rho(x)^{*}\right) d \lambda .
$$

Proof. Notice first that it follows from [13, 11.4.B] as in [5, (13.4.8)] that translates of functions in $L^{\mathrm{l}}(K) \cap P(K)$ belong to $\left[L^{\mathrm{l}}(K) \cap P(K)\right]$.

Therefore (and for linearity reasons) we can confine ourselves to the case $f \in L^{1}(K) \cap P(K)$ and $x=e$. Choose $u_{n} \in C_{00}^{+}(K)$ such that $\left\|u_{n}\right\|$ $=1$ and $\left\|f * u_{n} * u_{n}^{*}-f\right\|_{1} \rightarrow 0$. Since $f$ is continuous and $\|f\|_{\infty}=f(e)$, we can assume that

$$
f(e) \geq f * u_{n} * u_{n}^{*}(e) \text { and } f(e)=\lim f * u_{n} * u_{n}^{*}(e) .
$$

As $u_{n}^{*} * f * u_{n} \in P(K),[12,(D .39)]$ yields

$$
\begin{aligned}
0 & \leq \operatorname{tr} \rho\left(u_{n}^{*} * f * u_{n}\right) \leq \operatorname{tr}\left(\rho(f) \rho\left(u_{n} * u_{n}^{*}\right)\right) \\
& \leq \operatorname{tr} \rho(f)\left\|\rho\left(u_{n} * u_{n}^{*}\right)\right\| \leq \operatorname{tr} \rho(f) .
\end{aligned}
$$

Moreover, $|\operatorname{tr} \rho(g)| \leq d_{\rho}\|g\|_{1}$ holds for $g \in L^{1}(K)$. We define now $F$ and $F_{n}$ on $\hat{Z}$ by

$$
F(\lambda)=\sum_{\rho \in r^{-1}(\lambda)} k_{\rho} \operatorname{tr} \rho(f) \quad \text { and } \quad F_{n}(\lambda)=\sum_{\rho \in r^{-1}(\lambda)} k_{\rho} \operatorname{tr} \rho\left(f * u_{n} * u_{n}^{*}\right) .
$$

Let $\lambda \in \hat{Z}$ such that $F(\lambda)=\infty$. Then for $M>0$ we find $\rho_{1}, \ldots, \rho_{m} \in$ $r^{-1}(\lambda)$ so that $\sum_{l=1}^{m} k_{\rho_{l}} \operatorname{tr} \rho_{l}(f) \geq M+1$. It follows that for some $N \in \mathbf{N}$,

$$
\sum_{i=1}^{m} k_{\rho_{l}} \operatorname{tr} \rho_{l}\left(f * u_{n} * u_{n}^{*}\right) \geq M \text { for all } n \geq N,
$$

i.e. $F_{n}(\lambda) \rightarrow \infty$. If $F(\lambda)<\infty$, then for $\varepsilon>0$ there are $\rho_{1}, \ldots, \rho_{m} \in r^{-1}(\lambda)$ such that

$$
\sum_{\substack{\rho \in r^{-1}(\lambda) \\ \rho \neq \rho_{l}}} k_{\rho} \operatorname{tr} \rho(f)<\varepsilon .
$$

On the other hand, there exists $N \in \mathbf{N}$ so that

$$
\sum_{i=1}^{m} k_{\rho_{t}}\left(\operatorname{tr} \rho_{i}(f)-\operatorname{tr} \rho_{l}\left(f * u_{n} * u_{n}^{*}\right)\right)<\varepsilon
$$

for $n \geq N$; hence

$$
\begin{aligned}
0 & \leq F(\lambda)-F_{n}(\lambda) \\
& \leq \sum_{\substack{\rho \in r^{-1}(\lambda) \\
\rho \neq \rho_{l}}} k_{\rho} \operatorname{tr} \rho(f)+\sum_{i=1}^{m} k_{\rho_{t}}\left(\operatorname{tr} \rho_{i}(f)-\operatorname{tr} \rho_{i}\left(f * u_{n} * u_{n}^{*}\right)\right) \\
& <2 \varepsilon .
\end{aligned}
$$


Thus $F(\lambda)=\lim F_{n}(\lambda)$. Setting $G_{n}=\inf \left\{F_{k} ; k \geq n\right\}$, we get $0 \leq G_{n}(\lambda) \leq$ $F_{n}(\lambda), G_{n}(\lambda) \rightarrow F(\lambda)$ and, by Theorem 3.2,

$$
\begin{aligned}
\int_{\hat{Z}} G_{n}(\lambda) d \lambda & \leq \int_{\hat{Z}} F_{n}(\lambda) d \lambda=\left\langle f, u_{n}^{*} * u_{n}\right\rangle \\
& =f * u_{n} * u_{n}(e) \leq f(e) .
\end{aligned}
$$

[3, Ch. IV, §36, Theorem 5] now shows that $F \in L^{1}(\hat{Z})$ and

$$
\lim \int_{\hat{Z}} F_{n}(\lambda) d \lambda=\lim \int_{\hat{Z}} G_{n}(\lambda) d \lambda=\int_{\hat{Z}} F(\lambda) d \lambda .
$$

Finally, we obtain

$$
\begin{aligned}
f(e) & =\lim f * u_{n} * u_{n}^{*}(e)=\lim \int_{\hat{Z}} F_{n}(\lambda) d \lambda \\
& =\int_{\hat{Z}} F(\lambda) d \lambda=\int_{\hat{Z}} \sum_{\rho \in r^{-1}(\lambda)} k_{\rho} \operatorname{tr} \rho(f) d \lambda .
\end{aligned}
$$

4. Representations of double coset hypergroups and an example. Let $H$ be a compact subhypergroup of $K$ with normalized Haar measure $m_{H}$. The the double coset space $K / / H$ is again a hypergroup with convolution defined by

$$
\delta_{\dot{x}} * \delta_{\dot{y}}=\int_{H} \delta_{i} d \delta_{x} * m_{H} * \delta_{y}(t),
$$

where $\dot{z}=H z H$ for $z \in K[13, \S 14]$. Jewett $[13, \S 14]$ describes the connection between representations of $K$ and those of $K / / H$ by means of positive definite functions. For irreducible representations we need a more precise description.

For any representation $\pi$ of $K$, we will denote by $\dot{\pi}$ the corresponding representation of $K / / H$ defined by

$$
\dot{\pi}(\dot{x})=\pi\left(m_{H} * \delta_{x} * m_{H}\right) \text { for } x \in K .
$$

Lemma 4.1. Let $K$ and $H$ be as above. For $\pi \in \hat{K}$ the restriction of $\dot{\pi}$ to its essential subspace $K_{\dot{\pi}}$ is irreducible, and in this way one obtains every $\rho \in(K / / H) \hat{\text {. }}$

Proof. Notice first that $\dot{\pi} \neq 0$ yields $\pi\left(m_{H}\right) \neq 0$ and $\pi\left(m_{H}\right)$ is a hermitian projection. The subspace $E=\pi\left(m_{H}\right) H_{\pi}$ is $\dot{\pi}$-invariant, and $\dot{\pi} \mid E^{\perp}=0$. From $\dot{\pi}(\dot{x}) \eta=\pi\left(m_{H}\right) \pi(x) \eta$ for $\eta \in E$ it follows that every $\eta \neq 0$ is cyclic for $\dot{\pi} \mid E$. Thus $\dot{\pi} \mid E$ is irreducible and $E$ is the essential subspace $K_{\dot{\pi}}$ of $\dot{\pi}$. 


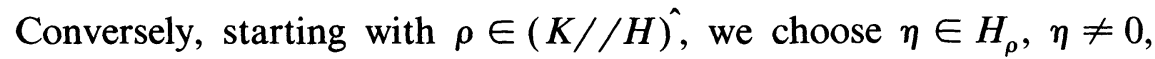
and define $\psi \in P(K / / H)$ by $\psi(\dot{x})=\langle\rho(\dot{x}) \eta, \eta\rangle . \psi$ is indecomposable and $\varphi \in P(K)$, defined by $\varphi(x)=\psi(\dot{x})$, as well. Hence there is a $\pi \in \hat{K}$ such that $\varphi(x)=\langle\pi(x) \xi, \xi\rangle$ for some $\xi \in H_{\pi}$ (compare $[13,11.3,11.4$ and 14.4]). From $\varphi(t)=1$ for every $t \in H$ it follows that $\pi(t) \xi=\xi$; hence $\pi\left(m_{H}\right) \xi=\xi$. Thus $\xi$ is a cyclic vector for $\dot{\pi} \mid K_{\dot{\pi}}$. Finally

$$
\langle\dot{\pi}(\dot{x}) \xi, \xi\rangle=\left\langle\pi\left(m_{H}\right) \pi(x) \xi, \xi\right\rangle=\int_{H} \varphi(t * x) d t=\psi(\dot{x})=\langle\rho(x) \eta, \eta\rangle
$$

holds; hence $\dot{\pi} \mid K_{\dot{\pi}}$ is equivalent to $\rho[13,11.4 \mathrm{D}]$.

REMARK 4.2. In a similar way we can obtain every cyclic representation of $K / / H$ as $\dot{\pi} \mid K_{\dot{\pi}}$ from some cyclic $\pi$ of $K$.

Every finite-dimensional irreducible representation $\pi$ of $K$ is uniquely determined by its normalized trace $\chi_{\pi}$ defined by

$$
\chi_{\pi}(x)=d_{\pi}^{-1} \cdot \operatorname{tr} \pi(x)
$$

for $x \in K$, and $\operatorname{dim} K_{\dot{\pi}}=\operatorname{tr} \pi\left(m_{H}\right)=\int_{H} \operatorname{tr} \pi(t) d t$ holds. If $\pi \mid H=$ $\bigoplus_{\rho \in \hat{H}} m_{\pi \mid H}(\rho) \cdot \rho$ is the decomposition of $\pi \mid H$ into irreducible representations, then

$$
\int_{H} \operatorname{tr} \pi(t) d t=\sum_{\rho \in \hat{H}} m_{\pi \mid H}(\rho) \int_{H} \operatorname{tr} \rho(t) d t=m_{\pi \mid H}(1)
$$

by the orthogonality relations. Thus $\dot{\pi} \neq 0$ iff $\int_{H} \operatorname{tr} \pi(t) d t \neq 0$, and for $\rho=\dot{\pi} \mid K_{\dot{\pi}}$ we have

$$
\operatorname{tr} \rho(\dot{x})=\operatorname{tr} \dot{\pi}(\dot{x})=\operatorname{tr}\left(\pi(x) \pi\left(m_{H}\right)\right)=\int_{H} \operatorname{tr} \pi(x * t) d t .
$$

Hence

$$
\chi_{\rho}(\dot{x})=\left(\int_{H} \chi_{\pi}(t) d t\right)^{-1} \cdot \int_{H} \chi_{\pi}(x * t) d t
$$

and $\int_{H} \chi_{\pi}(t) d t=d_{\pi}^{-1} \cdot m_{\pi}(1)$.

In particular, for a $Z$-hypergroup $K$ we get in view of Theorem 2.4:

COROLlaRY 4.3. $(K / / H)^{\hat{n}}$ can be identified with the set of normalized traces, i.e. with

$$
\left\{\dot{x} \rightarrow\left(\int_{H} \chi_{\pi}(t) d t\right)^{-1} \cdot \int_{H} \chi_{\pi}(x * t) d t ; \pi \in \hat{K}, \dot{\pi} \neq 0\right\} .
$$


We will now use this description to compute among others the irreducible representations of a certain non-commutative $Z$-hypergroup.

EXAMPLE 4.4. For a prime $p$, let $\Omega_{p}$ and $\Delta_{p}$ be the set of $p$-adic numbers (resp. $p$-adic integers) and $\Lambda_{k}=\left\{z \in \Omega_{p} ; z_{n}=0\right.$ for $\left.n<k\right\}$ $(k \in \mathbf{Z})$. Concerning the notations see $[12, \S 10]$. For $x_{i} \in \Delta_{p}(1 \leq i \leq 4)$ and $z \in \Omega_{p}$ we write $\left(x_{1}, x_{2}, x_{3}, x_{4}, z\right)$ for the matrix

$$
\left(\begin{array}{cccc}
1 & x_{1} & x_{2} & z \\
0 & 1 & 0 & x_{4} \\
0 & 0 & 1 & x_{3} \\
0 & 0 & 0 & 1
\end{array}\right),
$$

and denote by $G$ the group of these matrices. Clearly $G$ is a $Z$-group with center $\left\{(0,0,0,0, z) ; z \in \Omega_{p}\right\}$. Put $(t)=(t, 0,0,0,0)$ and $H=\{(t) ; t \in$ $\left.\Delta_{p}\right\}$. We are going to study the $Z$-hypergroup $K=G / / H$. First we compute it as a set, then determine the convolution, and finally describe the set of irreducible representations of $K$ in terms of the normalized traces.

(a) For $x=\left(x_{1}, x_{2}, x_{3}, x_{4}, z\right)$ and $y=\left(y_{1}, y_{2}, y_{3}, y_{4}, w\right) \in G$ we have

$$
\dot{x}=\dot{y} \quad \text { iff } \quad x_{i}=y_{i} \text { for } 2 \leq i \leq 4 \text { and } z-w \in x_{4} \cdot \Delta_{p} .
$$

Thus we can identify $K$ with the set

$$
\Delta_{p}^{2} \times \bigcup_{k=0}^{\infty}\left(\left(\Lambda_{k} \backslash \Lambda_{k-1}\right) \times\left(\Omega_{p} / \Lambda_{k}\right)\right)
$$

and we will write

$$
\dot{x}=\left(x_{2}, x_{3}, x_{4},[z]_{x_{4}}\right) \in K .
$$

(b) Since $G$ is a group the convolution on $K$ is given by

$$
\delta_{\dot{x}} * \delta_{\dot{y}}=\int_{H} \delta_{(x(t) y)} \cdot d t
$$

Now

$$
\begin{aligned}
(x(t) y)^{\cdot}=\left(x_{2}+y_{2},\right. & x_{3}+y_{3}, x_{4}+y_{4}, \\
& {\left.\left[z+w+x_{2} y_{3}+\left(t+x_{1}\right) y_{4}\right]_{x_{4}+y_{4}}\right) ; }
\end{aligned}
$$

hence

$$
\delta_{\dot{x}} * \delta_{\dot{y}}= \begin{cases}\delta_{(x y)^{*}} & \text { if } y_{4} \in\left(x_{4}+y_{4}\right) \Delta_{p}, \\ \int_{X} \delta_{\left(x_{2}+y_{2}, x_{3}+y_{3}, x_{4}+y_{4},\left[z+w+x_{2} y_{3}\right]_{x_{4}+y_{4}}+\dot{t}\right)} d \dot{t} & \text { otherwise }\end{cases}
$$


where $d \dot{t}$ denotes the normalized Haar measure on the compact group $X=y_{4} \Delta_{p} /\left(x_{4}+y_{4}\right) \Delta_{p}$. It follows at once that

$$
G(K)=\left\{\left(x_{2}, x_{3}, 0,[z]_{0}\right) ; x_{2}, x_{3} \in \Delta_{p}, z \in \Omega_{p}\right\}
$$

and

$$
Z=G(K) \cap Z(K)=\left\{\left(0,0,0,[z]_{0}\right) ; z \in \Omega_{p}\right\} .
$$

Notice that $G(K)$, and hence $K$, is non-abelian.

(c) Now the set $E(G)$ of normalized traces on $G$ can be easily computed, since $G$ is a nilpotent group of step 2. For $\chi \in E(G)$ we have $\chi \mid C \in \hat{C}$, where $C=\left\{(0,0,0,0, z) ; z \in \Omega_{p}\right\} \simeq \Omega_{p} . \hat{C}$ is the union of the annihilators $A\left(\hat{C}, \Lambda_{k}\right)$, which can be identified with $\Lambda_{-k+1} \subseteq \Omega_{p}$ by $w \rightarrow \lambda_{w}$, where $\lambda_{w}$ is defined by

$$
\lambda_{w}(z)= \begin{cases}\exp 2 \pi i\left(\sum_{n=m}^{k-1} z_{n} \cdot \sum_{s=n}^{k-1} p^{-s+n-1} \cdot w_{-s}\right) & \text { for } z \in \Lambda_{m}(m<k), \\ 1 & \text { otherwise }\end{cases}
$$

Now we put

$$
E_{0}(G)=\left\{\chi \in E(G) ; \chi \mid C \in A\left(\hat{C}, \Delta_{p}\right)\right\},
$$

and for $k>0$,

$$
E_{k}(G)=\left\{\chi \in E(G) ; \chi \mid C \in A\left(\hat{C}, \Lambda_{k}\right) \backslash A\left(\hat{C}, \Lambda_{k-1}\right)\right\} .
$$

For $G_{k}=\left\{x \in G ; x_{i} \in \Lambda_{k}\right.$ for $\left.1 \leq i \leq 4\right\}$ the following hold:

(i) $\chi \mid G \backslash G_{k}=0$ for $\chi \in E_{k}(G)$; and

(ii) $E_{k}(G) \mid G_{k} \simeq\left(\hat{\Lambda}_{k}\right)^{4} \times\left(A\left(\hat{C}, \Lambda_{k}\right) \backslash A\left(\hat{C}, \Lambda_{k-1}\right)\right)$ for $k>0$ and $E_{0}(G) \simeq\left(\hat{\Delta}_{p}\right)^{4} \times A\left(\hat{C}, \Delta_{p}\right)$.

Thus we have for $\lambda_{i} \in \hat{\Lambda}_{k}(1 \leq i \leq 4)$ and $w \in \Lambda_{-k+1} \backslash \Lambda_{-k+2}$ :

$$
\chi_{\left(\lambda_{1}, \lambda_{2}, \lambda_{3}, \lambda_{4}, w\right)}(x)= \begin{cases}\lambda_{w}(z) \prod_{i=1}^{4} \lambda_{i}\left(x_{i}\right) & \text { if } x \in G_{k}, \\ 0 & \text { otherwise. }\end{cases}
$$

We mention that, since the index of $G_{k}$ in $G$ is $p^{4 k}$, the dimension of a representation corresponding to an element of $E_{k}(G)$ is $p^{4 k}$.

Now it follows that

$$
\begin{aligned}
\int_{H} \chi_{\left(\lambda_{1}, \lambda_{2}, \lambda_{3}, \lambda_{4}, w\right)}(t, 0,0,0,0) d t & =p^{-k} \int_{\Lambda_{k}} \lambda_{1}(s) d s \\
& = \begin{cases}p^{-k} & \text { if } \lambda_{1}=1_{\Lambda_{k}}, \\
0 & \text { otherwise; }\end{cases}
\end{aligned}
$$


where $d s$ is the normalized Haar measure on $\Lambda_{k}$, which equals $p^{k} \cdot d t, d t$ the Haar measure on $H$.

Since $\left(x_{1}, x_{2}, x_{3}, x_{4}, z\right)(t, 0,0,0,0)=\left(x_{1}+t, x_{2}, x_{3}, x_{4}, z\right)$, we have

$$
\begin{aligned}
& \int_{H} \chi_{\left(\lambda_{1}, \lambda_{2}, \lambda_{3}, \lambda_{4}, w\right)}\left(x_{1}+t, x_{2}, x_{3}, x_{4}, z\right) d t \\
& \quad= \begin{cases}p^{-k} \int_{\Lambda_{k}} \lambda_{1}(s) d s \cdot \prod_{i=2}^{4} \lambda_{i}\left(x_{i}\right) \cdot \lambda_{w}(z) & \text { if } x_{i} \in \Lambda_{k}(i=2,3,4), \\
0 & \text { otherwise. }\end{cases}
\end{aligned}
$$

Thus $\hat{K}$ can be identified with

$$
\left(\left(\hat{\Delta}_{p}\right)^{3} \times \Lambda_{1}\right) \cup \bigcup_{k=1}^{\infty}\left(\left(\hat{\Lambda}_{k}\right)^{3} \times\left(\Lambda_{-k+1} \backslash \Lambda_{-k+2}\right)\right)
$$

$\operatorname{via}\left(\lambda_{2}, \lambda_{3}, \lambda_{4}, w\right) \rightarrow \chi_{\left(\lambda_{2}, \lambda_{3}, \lambda_{4}, w\right)}$, which is defined by

$$
\chi_{\left(\lambda_{2}, \lambda_{3}, \lambda_{4}, w\right)}\left(x_{2}, x_{3}, x_{4},[z]_{x_{4}}\right)= \begin{cases}\prod_{i=2}^{4} \lambda_{i}\left(x_{i}\right) \cdot \lambda_{w}(z), & x_{t} \in \Lambda_{k}, \\ 0 & \text { elsewhere. }\end{cases}
$$

Define $\hat{K}_{0}=r^{-1}\left(A\left(\hat{C}, \Delta_{p}\right)\right)$ and

$$
\hat{K}_{k}=r^{-1}\left(A\left(\hat{C}, \Lambda_{k}\right) \backslash A\left(\hat{C}, \Lambda_{k-1}\right)\right) \text { for } k>0,
$$

where $r: \hat{K} \rightarrow \hat{Z}$ is the mapping studied in $\S 2$. These subsets of $\hat{K}$ are both open and closed, and for the dimension of some $\rho \in \hat{K}_{k}$ we get

$$
d_{\rho}=\operatorname{dim} K_{\dot{\pi}}=d_{\pi} \cdot \int_{H} \chi_{\pi}(t) d t=p^{4 k} \cdot p^{-k}=p^{3 k}
$$

Thus the function $\rho \rightarrow d_{\rho}$ is continuous on $\hat{K}$ and the topology on $\hat{K}$ is that of uniform convergence on compact subsets of $K$.

\section{REFERENCES}

[1] R. J. Blattner, On induced representations, Amer. J. Math., 83 (1961), 79-98.

[2] __ Positive definite measures, Proc. Amer. Math. Soc., 14 (1963), 423-428.

[3] N. Bourbaki, Intégration, Ch. I-IV, Paris: Hermann 1952.

[4] A. K. Chilana and K. A. Ross, Spectral synthesis in hypergroups, Pacific J. Math., 76 (1978), 313-328.

[5] J. Dixmier, Les $C^{*}$-algèbres et leurs représentations, Paris: Gauthier-Villars 1964.

[6] Les algèbres d'opérateurs dans l'espace Hilbertien, Paris: Gauthier-Villars 1969.

[7] C. F. Dunkl, The measure algebra of a locally compact hypergroup, Trans. Amer. Math. Soc., 179 (1973), 331-348. 
[8] S. Grosser and M. Moskowitz, Representation theory of central topological groups, Trans. Amer. Math. Soc., 129 (1967), 361-390.

[9] Harmonic analysis on central topological groups, Trans. Amer. Math. Soc., 156 (1971), 419-455.

[10] S. Grosser, R. Mosak and M. Moskowitz, Duality and harmonic analysis on central topological groups, Indag. Math., 35 (1973), 65-91.

[11] R. W. Henrichs, Über Fortsetzung positiv definiter Funktionen, Math. Ann., 232 (1978), 131-150.

[12] E. Hewitt and K. A. Ross, Abstract Harmonic Analysis I, II, Berlin-Heidelberg-New York: Springer-Verlag, 1963/1970.

[13] R. I. Jewett, Spaces with an abstract convolution of measures, Advances in Math., 18 (1975), 1-101.

[14] H. Reiter, Classical Harmonic Analysis and Locally Compact Groups, Oxford: At the Clarendon Press 1968.

[15] K. A. Ross, Hypergroups and centers of measure algebras, Symposia Math., 22 (1977), 189-203.

[16] _ Centers of hypergroups, Trans. Amer. Math. Soc., 243 (1978), 251-259.

[17] R. Spector, Apercu de la Théorie des Hypergroupes, Lecture Notes in Mathematics 497 (Analyse harmonique sur les groupes de Lie, Sém. Nancy-Strasbourg 1973-1975), Springer-Verlag.

[18] _ Mesures invariantes sur les hypergroupes, Trans. Amer. Math. Soc., 239 (1978), 147-166.

[19] R. C. Vrem, Harmonic analysis on compact hypergroups, Pacific J. Math., 85 (1979), 239-251.

[20] P. S. Wang, On isolated points in the dual spaces of locally compact groups, Math. Ann., 218 (1975), 19-34.

Received March 31, 1982 and in revised form May 19, 1982. The third author was supported by a grant from the DAAD (German Academic Exchange Service) at the University of Paderborn.

Wilfried HaUENSCHILd AND EbERHARD KaNiUTH

FACHBEREICH MATHEMATIK/INFORMATIK

DER UNIVERSITÄT-GeSAMTHOCHSCHUle PADERBoRN

WARBURGER STRASSE 100

D-4790 PADERBORN

FEDERAL REPUBLIC OF GERMANY

AND

DEPARTMENT OF MATHEMATICS

UNIVERSITY OF DELHI

DELHI, 110007 INDIA 


\section{PACIFIC JOURNAL OF MATHEMATICS \\ EDITORS}

DONALD BABBITT (Managing Editor)

University of California

Los Angeles, CA 90024

Hugo RossI

University of Utah

Salt Lake City, UT 84112

C. C. MOOre and ARThur OGus

University of California

Berkeley, CA 94720
J. Dugundi

Department of Mathematics

University of Southern California

Los Angeles, CA 90089-1113

R. FINN and H. SAMELSON

Stanford University

Stanford, CA 94305

ASSOCIATE EDITORS

$\begin{array}{lllll}\text { R. Arens } & \text { E. F. Beckenbach } & \text { B. H. Neumann } & \text { F. Wolf } & \text { K. Yoshida }\end{array}$ $(1906-1982)$

\section{SUPPORTING INSTITUTIONS}

UNIVERSITY OF ARIZONA

UNIVERSITY OF BRITISH COLUMBIA

CALIFORNIA INSTITUTE OF TECHNOLOGY

UNIVERSITY OF CALIFORNIA

MONTANA STATE UNIVERSITY

UNIVERSITY OF NEVADA, RENO

NEW MEXICO STATE UNIVERSITY

OREGON STATE UNIVERSITY
UNIVERSITY OF OREGON

UNIVERSITY OF SOUTHERN CALIFORNIA

STANFORD UNIVERSITY

UNIVERSITY OF HAWAII

UNIVERSITY OF TOKYO

UNIVERSITY OF UTAH

WASHINGTON STATE UNIVERSITY

UNIVERSITY OF WASHINGTON 


\section{Pacific Journal of Mathematics}

\section{Vol. 110, No. $1 \quad$ September, 1984}

Wojciech Abramczuk, A class of surjective convolution operators $\ldots \ldots \ldots \ldots 1$

K. Adachi, Extending bounded holomorphic functions from certain

subvarieties of a weakly pseudoconvex domain $\ldots \ldots \ldots \ldots \ldots \ldots$

Malvina Florica Baica, An algorithm in a complex field and its application

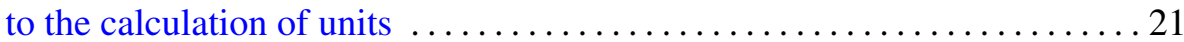

Giuliana Bianchi and Robert Cori, Colorings of hypermaps and a

conjecture of Brenner and Lyndon $\ldots \ldots \ldots \ldots \ldots \ldots \ldots \ldots \ldots \ldots \ldots \ldots$

Ronald James Evans, Determinations of Jacobsthal sums . . .......... 49

Leslie Foged, Characterizations of $\aleph$-spaces .................... 59

Nassif A. Ghoussoub and Paulette Saab, Weak compactness in spaces of

Bochner integrable functions and the Radon-Nikodým property . . . . . . 65

J. Gómez Gil, On local convexity of bounded weak topologies on Banach

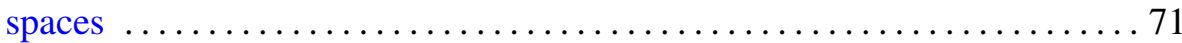

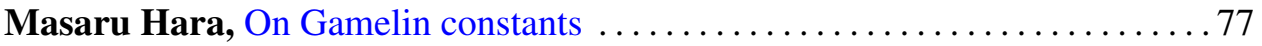

Wilfried Hauenschild, Eberhard Kaniuth and Ajay Kumar, Harmonic

analysis on central hypergroups and induced representations $\ldots \ldots \ldots 83$

Eugenio Hernandez, An interpolation theorem for analytic families of

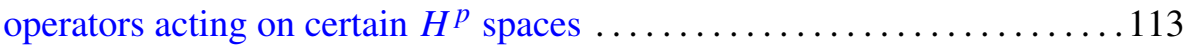

Thomas Alan Keagy, On "Tauberian theorems via block-dominated

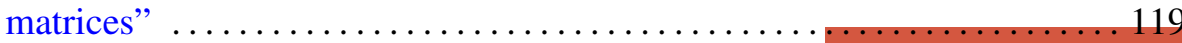

Thomas Landes, Permanence properties of normal structure $\ldots \ldots \ldots \ldots \ldots 125$

Daniel Henry Luecking, Closed ranged restriction operators on weighted

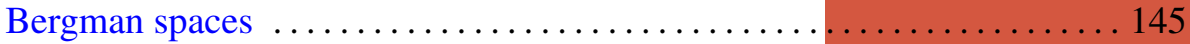

Albert Thomas Lundell, The $p$-equivalence of $\mathrm{SO}(2 n+1)$ and $\mathrm{Sp}(n) \ldots \ldots 161$

Mark D. Meyerson, Remarks on Fenn's "the table theorem" and Zaks' "the chair theorem" ..................................... 167

Marvin Victor Mielke, Homotopically trivial toposes . . . . . . . . . . 171

Gerard J. Murphy, Hyperinvariant subspaces and the topology on Lat A . . 183

Subhashis Nag, On the holomorphy of maps from a complex to a real manifold

Edgar Milan Palmer and Robert William Robinson, Enumeration of self-dual configurations ................................. 203

John J. Walsh and David Clifford Wilson, Continuous decompositions

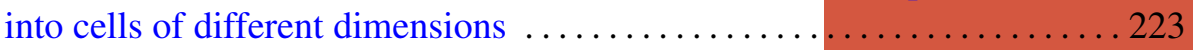

Walter John Whiteley, Infinitesimal motions of a bipartite framework .....233 